\title{
Scaling Transformation for Nonlocal Interactions
}

\author{
Hai-Jun Wang \\ Center for Theoretical Physics and School of Physics, Jilin University, Changchun, China \\ Email: whj@jlu.edu.cn
}

Received 22 March 2015; accepted 25 April 2015; published 28 April 2015

Copyright (C) 2015 by author and Scientific Research Publishing Inc.

This work is licensed under the Creative Commons Attribution International License (CC BY). http://creativecommons.org/licenses/by/4.0/

(c) (i) Open Access

\begin{abstract}
In the light of their relationships with renormalization, in this paper we associate the scaling transformation with nonlocal interactions. On one hand, the association leads us to interpret the nonlocality with locally symmetric method. On the other hand, we find that the nonlocal interaction between hadrons could be test ground for scaling transformation if ascribing the running effects in renormalization to scaling transformation. The nonlocal interaction Lagrangian turns out to vary under scaling transformation, analogous to running cases in renormalization. And the total Lagrangian becomes scale invariant only under some extreme conditions. The conservation law of this extreme Lagrangian is discussed and a contribution named scalum appears to the spin angular momentum. Finally a mechanism is designed to test the scaling effect on nonlocal interaction.
\end{abstract}

\section{Keywords}

\section{Scaling Transformation, Renormalization, Conservation Law, Spin Crisis, Nonlocal Scattering}

\section{Introduction}

Nowadays, on account of the developments of string theory [1] [2], Lattice QCD [3] and the necessity to describe nonperturbatively the intermediate strong interaction between extended hadrons [4], the construction of a consistent nonlocal theory is still called for [5]-[20]. The pioneering study of nonlocal interaction dates back to the 1930's [21] when quantum field theory was in its infancy. And the phenomenology of nonlocal interaction commenced with the primary attempts to describe the interaction between extended particles (such as hadrons [22] [23]), whilst to cope with the divergence appearing in local quantum field theories (LQFT). The development afterward purported mainly to give a consistently convergent theory in order to underlie the named "effective field theory”, whereof some form factors were usually employed [5]-[9] [24]-[36]. Whereas in such context one encounters the difficulty of unitarity and causality in formulating the S matrix [26] [36], no matter the 
Feldman-Yang [37] method or conventionally canonical quantization method [26]. Some promising progresses on this issue [6] [7] [9] [32] [33] [38]-[43] in one way or another showed their accordance with the renormalization methods [44]-[47].

In this paper we try to use the scaling transformation (dilatation of space-time), which is inspired by renormalization and assumed effective in nonlocal description of hadron physics, to unveil part of running effects in nonlocal interaction. More often than not, previous investigations on nonlocal interaction tried to fit certain results to those of renormalization [6] [9] [32] [33]. Reversely, in this paper we phenomenologically extract a scaling transformation from renormalization for nonlocal interaction based on their similar physical picture. With afterthought, the achievements of finding that nonlocal interaction is linked with renormalized interaction vertex may in one aspect owe to their common characteristic of effectively using form factors. For instance, in QED, the momentum-space vertex with renormalization is [48] [49]

$$
\Gamma^{\mu}\left(p^{\prime}, p\right)=\gamma^{\mu} F_{1}\left(q^{2}\right)+\frac{i \sigma^{\mu v} q_{v}}{2 m} F_{2}\left(q^{2}\right),
$$

where $q=p^{\prime}-p$, with $F_{1}\left(q^{2}\right)$ and $F_{2}\left(q^{2}\right)$ known as Dirac and Pauli form factors respectively. Similarly, the nonlocal interaction has its general form factors in coordinate-space [35] [36],

$$
L_{I}=-g \iint \mathrm{d}^{4} \xi \mathrm{d}^{4} \eta F(\xi, \eta) A_{\mu}(x) \bar{\psi}(x+\eta) \Gamma^{\mu} \psi(x+\xi) .
$$

Here the vertex $\Gamma^{\mu}$ could be the usual vector $\gamma^{\mu}$, tensor like $i \sigma^{\mu v} q_{v}$, or other forms to be determined. Its form in momentum-space then is $L_{I}=-g A_{\mu}\left(q^{2}\right) \bar{\psi}(p) F\left(p, p^{\prime}\right) \Gamma^{\mu} \psi\left(p^{\prime}\right)$, where $q=p^{\prime}-p, \psi\left(p^{\prime}\right)$ is the spinor in momentum space and the expansion like $\psi(x+\xi)=\sum_{\mathbf{p}} \psi(p) \mathrm{e}^{i p(x+\xi)}$ has been implicit. The $F\left(p, p^{\prime}\right)$ is defined as the Fourier transform of $F(\xi, \eta)$,

$$
F\left(p, p^{\prime}\right)=\iint \mathrm{d}^{4} \xi \mathrm{d}^{4} \eta \mathrm{e}^{i p \eta} F(\xi, \eta) \mathrm{e}^{-i p^{\prime} \xi} .
$$

The renormalization group method (RGM) has its intrinsic relationship with scaling transformation if viewing the differentiating operator $\mu \frac{\mathrm{d}}{\mathrm{d} \mu}$ in group equation as scaling operator. In RGM, for a function $\Lambda$ that represents a vertex function, a wave function or a propagator, its renormalized form and unrenormalized form are linked as [46]

$$
\Lambda=Z_{F} \Lambda_{R},
$$

whence the form factor $F\left(p, p^{\prime}\right)$ may be (approximately) viewed as just the collection of these $Z_{F}$ s, which are obtained by loop corrections. Differentiating the above equation with respect to renormalization parameter $\mu$, and in view of that unrenormalized $\Lambda$ is independent of $\mu$, one immediately gets

$$
\mu \frac{\mathrm{d} \Lambda_{R}}{\mathrm{~d} \mu}+\gamma_{F} \Lambda_{R}=0
$$

where $\gamma_{F}$ is the anomalous scaling dimension defined by

$$
\gamma_{F}=\mu \frac{\mathrm{d}}{\mathrm{d} \mu} \ln Z_{F} .
$$

In the next section one may note that the operator $\mu \frac{\mathrm{d}}{\mathrm{d} \mu}$ is just the scaling operator in its spatial representation, apart from a coefficient $i$. The Equation (4) is a special form of renormalization group equation, and the well known form is [50] [51]

$$
\left[M \frac{\partial}{\partial M}+\beta(\lambda) \frac{\partial}{\partial \lambda}+n \gamma(\lambda)\right] G^{(n)}\left(\left\{x_{i}\right\} ; M, \lambda\right)=0,
$$

which is for any Green's function of massless $\phi^{4}$ theory. Supposes the function $\Lambda_{R}$ has a dimension $\gamma_{F}$ 
with respect to a scale parameter $\mu$, then by such transformation $\mu \rightarrow \lambda \mu$, the $\Lambda_{R}$ yields

$$
\Lambda_{R}(\mu \text {, other parameters })=\lambda^{\gamma_{F}} \Lambda_{R}\left(\frac{\mu}{\lambda} \text {, other parameters }\right),
$$

that is the essence of RGM. Besides the obvious application of spatial scaling-transformation to the nonlocal form factor $F\left(p, p^{\prime}\right)$, in this paper we will focus on how it affects spinors consistently while involving its unitary-representation.

The scaling transformation, i.e. a freedom added to Poincare group to form Wely group [52], belongs to a larger group called 4-dimension Conformal Group, which in mathematical side has been investigated thoroughly from different aspects, and its application to physics especially to quantum field once was also widely considered. However the application is not so satisfactory because hitherto no other perfect quantum system than photon field [53] [54] has been found so that the corresponding Lagrangian is scaling invariant, i.e. demanding the mass of involved particles to be null [55]-[60]. Furthermore, one inference of the scale invariance is that according to Noether's theorem, if a Lagrangian is invariant under scaling transformation, then the trace of the energy-momentum tensor should be null [56] [58]. These two factors become obstacles to apply the scaling transformation to most material fields. Other efforts were also experienced to search for invariant fermion equation or scattering amplitude [57] [59] [61], and even to apply it to nonlocal action [62] [63]. None of the results is pertinent to known material fields. In this paper we investigate the application by trying two new tentative methods. One is to consider the unitary representation and the coordinate representation of conformal group simultaneously. The other is to apply the scale transformation to hadron physics since, the hadrons have their own sizes with which the interactions between them to some extent vary. Accordingly the test bed for conformal transformation might be nonlocal interaction between hadrons. However, here the scaling transformation works not for invariance, but for running. The running effects of nonlocal interaction are just like those in renormalization.

In this paper we shall use the scaling feature of RGM, but we free us from the detail calculation of renormalization. Since we are looking for a transformation method to interpret the running effect in nonlocal interaction, once we already got an effective form of Lagrangian or Hamiltonian, we would just use tree-level form to do calculations. The further loop calculation will double count something, Born approximation is fine for most cases of interest. The calculation resembles that used in deep inelastic scattering, though we are involved just elastic processes. In summary, in the whole paper we focus more on the properties of scaling transformation/conformal group, and on how to apply them to nonlocal fields.

The rest of the paper is arranged as follows. Sect. II is dedicated to introducing the two representations of scale transformation, i.e. the coordinate/operator representation and spinor/unitary representation. In Sect. III we establish the physical relationship between the two representations, on condition that a scale invariant vertex exists. Subsequently in Sect. IV we discuss the conservation law for the derived scaling-invariant vertex, and the possibility that it relates to the nucleon's polarizations is posed. In Sect. V according to the characteristics of applying the general vertex-form $\gamma^{\mu}\left(a+b \gamma^{5}\right)$ to polarized scattering, a mechanism is proposed to examine the predictions on nonlocality. Conclusions and discussions are presented finally.

\section{The Spatial and Spinor Representations for Scaling Transformation Based on Group Theory}

It is well known that the scaling transformation belongs to a larger Conformal Group [52] [56], therefore next we will learn first the properties of 4-dimensional Conformal Group, including its spatial/operator representation and unitary/spinor representation, as well as commutations among their generators. At the end of this section, we

will understand the role of operator $\mu \frac{\mathrm{d}}{\mathrm{d} \mu}$ in the conformal group. The spatial representation is mainly referencing to that of Ref. [52] [64] and the unitary representation is derived by applying Cartan method [65] to $S O(6)-S U(4)$ transform. The unitary representation is the focus of this section, and of this paper as well.

Mostly the scaling transformation in 4-dimension is discussed as a subset of conformal group, and in previous literature its applications are seldom considered independently [56]. Here we start with the null vector space (Euclidean space), 


$$
\eta_{1}^{2}+\eta_{2}^{2}+\eta_{3}^{2}+\eta_{4}^{2}+\eta_{5}^{2}+\eta_{6}^{2}=0
$$

reserving which gives the popular definition of conformal group [65]. A special expression of the differential forms in 4-dimension spatial representation can be derived directly from the above equation. In derivation we need to apply the following variables [52]

$$
x_{\mu}=\frac{\eta_{\mu}}{K}, \text { where } K=\eta_{5}+i \eta_{6} \text {, where } \mu=1,2,3,4
$$

together with the differential form

$$
\frac{\partial}{\partial \eta_{a}}=\frac{1}{K}\left\{\left[\delta_{a \mu}-\left(\delta_{a 5}+i \delta_{a 6}\right) x_{\mu}\right] \frac{\partial}{\partial x_{\mu}}+\left(\delta_{a 5}+i \delta_{a 6}\right) K \frac{\partial}{\partial K}\right\} \text {, where } a=1,2, \cdots, 6
$$

to the definition of 6-dimensional angular-momentum

$$
M_{a b}=i\left(\eta_{a} \frac{\partial}{\partial \eta_{b}}-\eta_{b} \frac{\partial}{\partial \eta_{a}}\right), \text { where } a, b=1,2, \cdots, 6 .
$$

Then one gets the following generators for conformal group [52] [of which in Equation (56)]

$$
\begin{aligned}
& D=i M_{56}=-\left(\eta_{5} \frac{\partial}{\partial \eta_{6}}-\eta_{6} \frac{\partial}{\partial \eta_{5}}\right)=i\left(x_{\mu} \frac{\partial}{\partial x_{\mu}}-K \frac{\partial}{\partial K}\right), P_{\mu}=M_{5 \mu}+i M_{6 \mu}=i \frac{\partial}{\partial x_{\mu}}, \\
& K_{\mu}=M_{5 \mu}-i M_{6 \mu}=i\left\{-x^{2} \frac{\partial}{\partial x_{\mu}}+2 x_{\mu} x_{v} \frac{\partial}{\partial x_{v}}-2 K x_{\mu} \frac{\partial}{\partial K}\right\},
\end{aligned}
$$

The projected form (making $K$ as constant boundary of Minkowski Space [66]) with Minkowski convention then is

$$
D=i x_{\mu} \frac{\partial}{\partial x_{\mu}}, M_{\mu v}=i\left(x_{\mu} \frac{\partial}{\partial x^{v}}-x_{v} \frac{\partial}{\partial x^{\mu}}\right), P_{\mu}=i \frac{\partial}{\partial x^{\mu}}, K_{\mu}=-i\left(x^{2} \frac{\partial}{\partial x^{\mu}}-2 x_{\mu} x^{v} \frac{\partial}{\partial x^{v}}\right),
$$

where $M_{\mu v}$ represent the components of conventional angular momentum in 4-dimension. The corresponding commutation relation can be obtained by direct computation,

$$
\begin{aligned}
& {\left[M_{\mu v}, M_{\rho \sigma}\right]=i\left(g_{v \rho} M_{\mu \sigma}+g_{\mu \sigma} M_{v \rho}-g_{\mu \rho} M_{v \sigma}-g_{v \sigma} M_{\mu \rho}\right),} \\
& {\left[M_{\mu v}, P_{\rho}\right]=i\left(g_{v \rho} P_{\mu}-g_{\mu \rho} P_{v}\right),\left[D, P_{\mu}\right]=-i P_{\mu},\left[D, K_{\mu}\right]=i K_{\mu},\left[D, M_{\mu v}\right]=0, \cdots}
\end{aligned}
$$

Before using Cartan method to achieve the unitary representation of Conformal Group, let's review first the steps of Cartan method with $S O(3)-S U(2)$ mapping as an example [65] (of which in pp. 41-48). To keep the invariance of $x_{1}^{2}+x_{2}^{2}+x_{3}^{2}=0$, one defines the matrix

$$
X=\left(\begin{array}{cc}
x_{3} & x_{1}-i x_{2} \\
x_{1}+i x_{2} & -x_{3}
\end{array}\right)
$$

The trace $\operatorname{Tr}\left(X^{\dagger} X\right)$ is $x_{1}^{2}+x_{2}^{2}+x_{3}^{2}$. With $U$ as an element of $S U(2)$ group, we define

$$
X^{\prime}=U^{-1} X U,
$$

immediately we have

$$
\operatorname{Tr}\left(X^{\prime \dagger} X^{\prime}\right)=\operatorname{Tr}\left(X^{\dagger} X\right)
$$

thus $S U(2)$ group keeps the trace invariant, and by this way the group also keeps the metric $x_{1}^{2}+x_{2}^{2}+x_{3}^{2}$. With the knowledge that the $S O(3)$ group directly reserves the metric $x_{1}^{2}+x_{2}^{2}+x_{3}^{2}$, we conclude that Cartan 
matrix $X$ acts as a mapping between $S O(3)$ and $S U(2)$. By the Cartan Matrix $X$, one can define spinor $\left(\begin{array}{l}\xi_{0} \\ \xi_{1}\end{array}\right)$ by

$$
X\left(\begin{array}{l}
\xi_{0} \\
\xi_{1}
\end{array}\right)=0
$$

with the solution $\xi_{0}= \pm \sqrt{\frac{x_{1}-i x_{2}}{2}}$ and $\xi_{1}= \pm \sqrt{\frac{-x_{1}-i x_{2}}{2}}$, and the reverse yields

$$
x_{1}=\xi_{0}^{2}-\xi_{1}^{2}, x_{2}=i\left(\xi_{0}^{2}+\xi_{1}^{2}\right), x_{3}=-2 \xi_{0} \xi_{1},
$$

which automatically satisfies $x_{1}^{2}+x_{2}^{2}+x_{3}^{2}=0$ from which we can define the spinor reversely.

From the above Cartan matrix $X$ we can extract the Pauli matrices $\sigma_{1}, \sigma_{2}, \sigma_{3}$ separately from the coefficients of $x_{1}, x_{2}, x_{3}$. Meanwhile Pauli matrices $\sigma_{1}, \sigma_{2}, \sigma_{3}$ act as the generators of $S U(2)$ group mentioned above. Furthermore it is easy to test that $S U(2)$ group reserves the metric

$$
\left|\xi_{0}\right|^{2}+\left|\xi_{1}\right|^{2}=\Xi^{\dagger} \Xi
$$

And coincidentally the $n$-vectors form (defined in Equation (24)) based on Pauli matrices don't generate new matrices, neither the multiplications nor the commutations among them, they themselves are closed. Now in what follows we would find the corresponding Cartan matrix from $S O(6)$ to $S U(4) / S U(2,2)$, namely the spinor representation for 4-dimension Conformal group.

To achieve its unitary/spinor representation in 4-dimension, mimicking the relationship between the metric $x_{1}^{2}+x_{2}^{2}+x_{3}^{2}$ and that in Equation (17), we shall associate the metric in Equation (5) with the invariant quadratic form

$$
\left|z_{1}\right|^{2}+\left|z_{2}\right|^{2}+\left|z_{3}\right|^{2}+\left|z_{4}\right|^{2}=Z^{\dagger} Z,
$$

by the following matrix [67],

$$
A=\left(\begin{array}{cccc}
0 & x_{1}+i x_{2} & x_{3}+i x_{4} & x_{5}+i x_{6} \\
-\left(x_{1}+i x_{2}\right) & 0 & x_{5}-i x_{6} & -x_{3}+i x_{4} \\
-\left(x_{3}+i x_{4}\right) & -x_{5}+i x_{6} & 0 & x_{1}-i x_{2} \\
-\left(x_{5}+i x_{6}\right) & x_{3}-i x_{4} & -x_{1}+i x_{2} & 0
\end{array}\right)
$$

Count the degrees of freedom of the groups that conserve separately Equation (5) and Equation (18), one finds they are both 15 . Next we only need to extract the coefficients before $x_{i}$ 's to get the unitary matrices as generators of $S U(4)$, just like the method used in three dimension example Equations (11)-(16). If we want to get the generators of $S U(2,2)$ we need only to change the signs before $x_{1}$ and $x_{2}$ and those ahead of corresponding matrices, which would change the Equations (5) and (18) to

$$
-x_{1}^{2}-x_{2}^{2}+x_{3}^{2}+x_{4}^{2}+x_{5}^{2}+x_{6}^{2}=0 .
$$

and

$$
-\left|z_{1}\right|^{2}-\left|z_{2}\right|^{2}+\left|z_{3}\right|^{2}+\left|z_{4}\right|^{2}=Z^{\dagger} Z .
$$

the latter falls into Dirac spinor like

$$
\tilde{\psi}=\left(z_{1}, z_{2}, z_{3}, z_{4}\right) .
$$

It can be examined that the matrix $A$ in Equation (19) meets the invariant expression

$$
\operatorname{Tr}\left(A^{\dagger} A\right)=4\left(x_{1}^{2}+x_{2}^{2}+x_{3}^{2}+x_{4}^{2}+x_{5}^{2}+x_{6}^{2}\right),
$$


just like the above 3-dimension example, while the $S U(4)$ group keeps the above trace

$$
x_{1}^{2}+x_{2}^{2}+x_{3}^{2}+x_{4}^{2}+x_{5}^{2}+x_{6}^{2}=\text { constant },
$$

it simultaneously reserves the metric Equation (18). The above method of linking real metric to a matrix is closely analogous to the Cartan method of constructing a spinor representation in any real space. Actually, the true spinor space for 4-d conformal group following Cartan method should be of 8-dimension instead of 4-dimension [65] [of which in pp. 88-89]. In what follows we would take over the process of deriving all of the $n$-vectors along the Cartan method [65] [of which in pp. 81-83], though we work in 4-dimension rather than 8-dimension. First we extract the matrices before $x_{i}$ 's in Equation (19), i.e. 1-vectors,

$$
\begin{aligned}
& B_{1}=\left(\begin{array}{cc}
i \sigma_{2} & 0 \\
0 & i \sigma_{2}
\end{array}\right), B_{2}=\left(\begin{array}{cc}
-\sigma_{2} & 0 \\
0 & \sigma_{2}
\end{array}\right), B_{3}=\left(\begin{array}{cc}
0 & \sigma_{3} \\
-\sigma_{3} & 0
\end{array}\right) ; \\
& B_{4}=\left(\begin{array}{cc}
0 & i I \\
-i I & 0
\end{array}\right), B_{5}=\left(\begin{array}{cc}
0 & \sigma_{1} \\
-\sigma_{1} & 0
\end{array}\right), B_{6}=\left(\begin{array}{cc}
0 & -\sigma_{2} \\
-\sigma_{2} & 0
\end{array}\right) .
\end{aligned}
$$

where $\sigma_{i}$ 's are Pauli matrices. The definition of $k$-vector is

$$
B_{k \text {-vector }}=\sum_{P}(-1)^{P} B_{n_{1}} B_{n_{2}} \cdots B_{n_{k}} \text {, }
$$

where $P$ denotes different permutations. Apply the above formula to 2-vector, and use the corresponding subscripts to denote the 1-vectors involved, then

$$
B_{12}=B_{1} B_{2}-B_{2} B_{1}=\left(\begin{array}{cc}
i \sigma_{2} & 0 \\
0 & i \sigma_{2}
\end{array}\right)\left(\begin{array}{cc}
-\sigma_{2} & 0 \\
0 & \sigma_{2}
\end{array}\right)-\left(\begin{array}{cc}
-\sigma_{2} & 0 \\
0 & \sigma_{2}
\end{array}\right)\left(\begin{array}{cc}
i \sigma_{2} & 0 \\
0 & i \sigma_{2}
\end{array}\right)=0 .
$$

Similarly, let's exhaust all possibilities, then obtain other nontrivial 2-vectors

$$
\begin{aligned}
B_{13} & =2\left(\begin{array}{cc}
0 & -\sigma_{1} \\
\sigma_{1} & 0
\end{array}\right), B_{15}=2\left(\begin{array}{cc}
0 & \sigma_{3} \\
-\sigma_{3} & 0
\end{array}\right), B_{35}=2 i\left(\begin{array}{cc}
\sigma_{2} & 0 \\
0 & \sigma_{2}
\end{array}\right), B_{36}=2 i\left(\begin{array}{cc}
\sigma_{1} & 0 \\
0 & -\sigma_{1}
\end{array}\right), \\
B_{46} & =-2 i\left(\begin{array}{cc}
\sigma_{2} & 0 \\
0 & -\sigma_{2}
\end{array}\right), B_{24}=2 i\left(\begin{array}{cc}
0 & \sigma_{2} \\
\sigma_{2} & 0
\end{array}\right), B_{23}=-2 i\left(\begin{array}{cc}
0 & \sigma_{1} \\
\sigma_{1} & 0
\end{array}\right) .
\end{aligned}
$$

We note that the new ones which are independent of $B_{i}$ 's are just $B_{23}$ and $B_{36}$. The same line can be followed to carry out the 3 -vectors. Ignoring the repeating ones, we find the new 3 -vectors independent of both 1 -vectors and 2-vectors are

$$
\begin{aligned}
& B_{123} \sim\left(\begin{array}{cc}
0 & \sigma_{3} \\
\sigma_{3} & 0
\end{array}\right), B_{134} \sim\left(\begin{array}{cc}
\sigma_{1} & 0 \\
0 & \sigma_{1}
\end{array}\right), B_{145} \sim\left(\begin{array}{cc}
\sigma_{3} & 0 \\
0 & \sigma_{3}
\end{array}\right), B_{245} \sim\left(\begin{array}{cc}
\sigma_{3} & 0 \\
0 & -\sigma_{3}
\end{array}\right), \\
& B_{345} \sim\left(\begin{array}{cc}
0 & \sigma_{2} \\
-\sigma_{2} & 0
\end{array}\right), B_{146} \sim\left(\begin{array}{cc}
I & 0 \\
0 & -I
\end{array}\right), B_{124} \sim\left(\begin{array}{ll}
0 & I \\
I & 0
\end{array}\right) .
\end{aligned}
$$

Computing the 4-vectors and the higher ones would not give new independent matrices. Finally, we can rearrange all above k-vector-produced matrices as follows [67],

$$
U_{i}=\frac{1}{2}\left(\begin{array}{cc}
\sigma_{i} & 0 \\
0 & \sigma_{i}
\end{array}\right), V_{\mu}=-\frac{1}{2}\left(\begin{array}{cc}
\sigma_{\mu} & 0 \\
0 & -\sigma_{\mu}
\end{array}\right), W_{\mu}=\frac{i}{2}\left(\begin{array}{cc}
0 & \sigma_{\mu} \\
\sigma_{\mu} & 0
\end{array}\right), Y_{\mu}=\frac{1}{2}\left(\begin{array}{cc}
0 & \sigma_{\mu} \\
-\sigma_{\mu} & 0
\end{array}\right),
$$

where $\sigma_{i}, i=1,2,3$ are normal Pauli matrices and $\sigma_{0}=\left(\begin{array}{ll}1 & 0 \\ 0 & 1\end{array}\right)$. The convention can be changed from Minkowski to Euclidean spaces while instead requiring $\sigma_{\mu}^{2}=-1$, i.e. making $\sigma_{0}=i$ and replacing definition of $\sigma_{i}$ by those in [67].

The route of inquiring the concrete matrices following Cartan method as above could be a shortcut that rarely 
mentioned in literature. It is can be checked that the commutations among $U_{i}, V_{\mu}, W_{\mu}, Y_{\mu}$ are just those for conformal group [52] [54], accordingly the mapping from these matrices to corresponding differential-forms turns out to be

$$
\begin{aligned}
& U_{i} \leftrightarrow \gamma_{i} \gamma_{j} \rightarrow i\left(x_{j} \frac{\partial}{\partial x^{k}}-x_{k} \frac{\partial}{\partial x^{j}}\right) \rightarrow M_{j k}, \\
& W_{i} \leftrightarrow \gamma_{0} \gamma_{i} \rightarrow i\left(x_{i} \frac{\partial}{\partial x^{0}}-x_{0} \frac{\partial}{\partial x^{i}}\right) \rightarrow M_{0 k}, \\
& W_{0} \leftrightarrow \gamma_{5} \rightarrow i x_{\mu} \frac{\partial}{\partial x_{\mu}} \rightarrow D, \\
& V_{\mu}+Y_{\mu} \leftrightarrow \gamma_{\mu}\left(1 \pm \gamma_{5}\right) \rightarrow i \frac{\partial}{\partial x^{\mu}} \rightarrow P_{\mu}, \\
& V_{\mu}-Y_{\mu} \leftrightarrow \gamma_{\mu}\left(1 \mp \gamma_{5}\right) \rightarrow-i\left(\frac{1}{2} x_{v} x^{\nu} \frac{\partial}{\partial x_{\mu}}-x_{\mu} x_{v} \frac{\partial}{\partial x_{v}}\right) \rightarrow K_{\mu} .
\end{aligned}
$$

We use $\rightarrow$ to represent the accurate mappings and $\leftrightarrow$ the equivalence, and the commutations have been examined by computer. Now we recognize that the role of operator $\mu \frac{\mathrm{d}}{\mathrm{d} \mu}$ (or $x_{\mu} \frac{\partial}{\partial x_{\mu}}$ ) in the conformal group is equivalent to the scaling operator $D$, with its unitary form $\gamma_{5}$.

\section{The Physical Relationship between the Two Representations of Scaling Transformation}

Enlightened by Lorentz transformation, in this section we try to link physically the spatial form of scaling transformation with its spinor/unitary form, the former representing the realistic expansions and contractions of space-time (dilatation and shrinkage means the same), the latter representing the intrinsic freedom very like spin angular momentum. Considering both representations in a sole frame is the main feature of this paper.

As for a nonlocal interaction $F\left(q^{2}\right) A^{v}\left(q^{2}\right) \bar{\psi}(p) \gamma_{\nu} \psi\left(p^{\prime}\right)$, besides knowing that the form factor $F\left(q^{2}\right)$ runs with scaling parameter as described Equation in (4), we are also curious about how a nonlocal interaction vertex $\gamma_{\mu}$ varies with scale. Before drawing any conclusion, let's first find the invariant vertex $\Gamma_{\mu}$ under the scaling transformation by mimicking the method of utilizing Lorentz transformation to Dirac equation. In this way we link its spatial form with its spinor form. As for Lorentz transformation, the transformation matrix $\left(\Lambda^{v}{ }_{\mu}\right)$ for $j^{\mu}(y)=\bar{\psi}(y) \gamma^{\mu} \psi(y)$ corresponds to a complex transformation $S$ for $\psi(y)$ so that the effect of the transformed result $\bar{\psi}(y) S^{-1} \gamma^{\mu} S \psi(y)$ is equivalent to $\bar{\psi}(y) \Lambda^{\mu}{ }_{v} \gamma^{\nu} \psi(y)$. Referencing the case of Lorentz transformation, our goal in this section is to search for the corresponding vertex-form $\Gamma^{\mu}$ so that it links with transformation $S^{\prime}$ by $S^{\prime-1} \Gamma^{\mu} S^{\prime}=\Lambda_{v}^{\prime \mu} \Gamma^{v}$, where $S^{\prime}=\mathrm{e}^{\frac{u}{2} \gamma_{5}}, \gamma_{5}$ is the spinor representation of the scaling operator $D$, and $\Lambda_{v}^{\prime \mu}$ represent tensor's components of scaling transformation.

Usually we perform the spatial Lorentz transformation on the vectors $A_{\mu}$ and $\gamma^{\mu}$. Obviously this combination brings about invariant formalism like $A^{v}\left(q^{2}\right) \bar{\psi}(p) \gamma_{\nu} \psi\left(p^{\prime}\right)$. We follow the convention that the same set of $\left\{\gamma^{\mu}\right\}$ is used in different coordinate systems, which naturally yields an equivalence transformation $S$ satisfying [68] [69]

$$
S^{-1} \gamma^{\mu} S=\Lambda_{v}^{\mu} \gamma^{v}=\gamma^{\prime \mu}
$$

where $\Lambda_{v}^{\mu}$ stand for the tensors' components of the Lorentz transformation. Substituting the Equation (29) into $A_{\mu}(x) \bar{\psi}(x) \gamma^{\mu} \psi(x)$ yields

$$
A_{\mu}^{\prime}(y) \bar{\psi}^{\prime}(y) S^{-1} \gamma^{\mu} S \psi^{\prime}(y)=A_{\mu}^{\prime}(y) \bar{\psi}(y) \gamma^{\prime \mu} \psi(y) .
$$

While looking for $\Gamma^{\mu}$ we would follow the same convention as that in the above paragraph, i.e., in different coordinate system we use the same set of $\left\{\Gamma^{\mu}\right\}$. Then analogously, we use the form of the above formula Equation (29) for scaling transformation as 


$$
S^{\prime-1} \Gamma^{\mu} S^{\prime}=\Lambda_{v}^{\prime \mu} \Gamma^{v},
$$

where formally we have used $\Lambda_{v}^{\prime \mu}$ to represent the scaling transformation to every coordinate component [57] [59] [61] [of which Equation (2)] instead of using the usual form $\mathrm{e}^{-\alpha}$ [64]. Slightly different from the operator $\mu \frac{\mathrm{d}}{\mathrm{d} \mu}$ appearing in renormalization group equation, here the operator $D$ has the usual form $D=i x^{v} \partial_{v}$, being a hermit one. With the relation $\mathrm{e}^{-i \alpha D} p_{\mu} \mathrm{e}^{i \alpha D}=\mathrm{e}^{-\alpha} p_{\mu}$, i.e. $\left[D, p_{\mu}\right]=-i p_{\mu}$ [64], we have

$$
\left(\Gamma^{\mu} p_{\mu}\right)_{\text {scaling transform }}^{\prime}=S^{\prime-1} \Gamma^{\mu} S^{\prime} \Lambda_{\mu}^{\prime \nu} p_{v}=S^{\prime-1} \Gamma^{\mu} S^{\prime} \mathrm{e}^{-i \alpha D} p_{\mu} \mathrm{e}^{i \alpha D} \text {. }
$$

Now let's substitute $S^{\prime}=\mathrm{e}^{\frac{u}{2} \gamma_{5}}$ obtained from the last section, where $u$ is the infinitesimal parameter. Formally we get

$$
\begin{aligned}
S^{\prime-1} \Gamma^{\mu} S^{\prime} \Lambda^{\prime \prime}{ }_{\mu} p_{v} & =\mathrm{e}^{-\frac{u}{2} \gamma_{5}} \Gamma^{\mu} \mathrm{e}^{\frac{u}{2} \gamma_{5}}\left(p_{\mu}\right)_{\text {scaling transform }}^{\prime}=\mathrm{e}^{-\frac{u}{2} \gamma_{5}} \Gamma^{\mu} \mathrm{e}^{\frac{u}{2} \gamma_{5}} \mathrm{e}^{-i \alpha D} p_{\mu} \mathrm{e}^{i \alpha D} \\
& =\mathrm{e}^{-\frac{u}{2} \gamma_{5}} \Gamma^{\mu} \mathrm{e}^{\frac{u}{2} \gamma_{5}} \mathrm{e}^{-\alpha} p_{\mu} \doteq \mathrm{e}^{-\frac{u}{2} \gamma_{5}} \Gamma^{\mu} \mathrm{e}^{\frac{u}{2} \gamma_{5}} p_{\mu}(1-\alpha) .
\end{aligned}
$$

From the experience of calculating $\gamma$-matrix and the following relations

$$
\begin{aligned}
& \mathrm{e}^{-\frac{u}{2} \gamma_{5}} \gamma^{\mu} \mathrm{e}^{\frac{u}{2} \gamma_{5}} \simeq\left(1-\frac{u}{2} \gamma_{5}\right) \gamma^{\mu}\left(1+\frac{u}{2} \gamma_{5}\right) \simeq \gamma^{\mu}+u \gamma^{\mu} \gamma_{5}, \\
& \mathrm{e}^{-\frac{u}{2} \gamma_{5}} \gamma^{\mu} \gamma_{5} \mathrm{e}^{\frac{u}{2} \gamma_{5}} \simeq\left(1-\frac{u}{2} \gamma_{5}\right) \gamma^{\mu} \gamma_{5}\left(1+\frac{u}{2} \gamma_{5}\right) \simeq \gamma^{\mu} \gamma_{5}+u \gamma^{\mu}, \\
& \mathrm{e}^{-\frac{u}{2} \gamma_{5}} \gamma^{\mu}\left(1 \pm \gamma_{5}\right) \mathrm{e}^{\frac{u}{2} \gamma_{5}} \simeq\left(1-\frac{u}{2} \gamma_{5}\right) \gamma^{\mu}\left(1 \pm \gamma_{5}\right)\left(1+\frac{u}{2} \gamma_{5}\right) \simeq(1 \pm u) \gamma^{\mu}\left(1 \pm \gamma_{5}\right),
\end{aligned}
$$

we find out a possible form of $\Gamma^{\mu}$

$$
\Gamma^{\mu}=\gamma^{\mu}\left(1 \pm \gamma_{5}\right) \text { while } \alpha \sim u \text {. }
$$

The coefficients $(1 \pm u)$ of Equation (36) can be contracted now to be 1 with coefficients $(1 \mp u)$ that come from the transformation of $p_{\mu}$. And we note that the infinitesimal parameters $u$ and $\alpha$ are not independent. By this way we set up the relationship between the operator $D$ and its unitary counterpart $S^{\prime}=\mathrm{e}^{\frac{u}{2} \gamma_{5}}$ directly. The key element of linking the two operators $D$ and $S^{\prime}=\mathrm{e}^{\frac{u}{2} \gamma_{5}}$ is the scale-invariant vertex $\Gamma^{\mu}$. One notes that $S^{\prime}=\mathrm{e}^{\frac{u}{2} \gamma_{5}}$ is responsible for acting on Dirac spinor as expected, or equivalently on the vertex $\Gamma^{\mu}$. And the operator $D$ is responsible for acting on the real vector coupling to $\Gamma^{\mu}$. Thus the scaling invariance holds true for interaction vertex $\Gamma^{\mu} A_{\mu}$, as well as for $\Gamma^{\mu} p_{\mu}$. The resultant vertex $\Gamma^{\mu}=\gamma^{\mu}\left(1 \pm \gamma_{5}\right)$ is different from that of Ref. [61] due to the choice of $\gamma_{5}$, since we have followed the convention of Quantum Field Theory. All in all, we have extended transformation, interaction vertex and spinor space simultaneously, which is reasonable from the viewpoint of entirety.

Now we are interested in what if we perform the scaling transformation $S^{\prime}$ succeedingly $N$ times upon the vector vertex-form $\gamma^{\mu}$. How the vector vertex form $\gamma^{\mu}$ varies with scaling is the starting point as well as the end of this research. Different from Equations (34)-(36), now we employ the following formulism without approximation

$$
\left(\mathrm{e}^{-\frac{u}{2} \gamma_{5}}\right)^{N} \gamma^{\mu}\left(\mathrm{e}^{\frac{u}{2} \gamma_{5}}\right)^{N}=\gamma^{\mu} \cosh N u+\gamma^{\mu} \gamma_{5} \sinh N u,
$$


from which one notes that the vector vertex arrives at its limits $\gamma^{\mu}\left(1 \pm \gamma_{5}\right)$ only if $\frac{\cosh N u}{\sinh N u} \rightarrow \pm 1$, i.e. $\mathrm{Nu} \rightarrow \pm \infty . \mathrm{Nu} \rightarrow \pm \infty$ means one carrying out enough steps of inflating or shrinking transformation. We call such states that involve interaction vertices $\gamma^{\mu}\left(1 \pm \gamma_{5}\right)$ as extreme states, which evolve from the interaction vertex $\gamma^{\mu}$ after the scale constantly changing. And the variation of coupling constant is assumed to be absorbed into $F\left(q^{2}\right)$. It turns out that such scaling transformation doesn't conserve the vector-dominant interaction, or alternatively, the transformation tends to transform the relating spinor from a normal one to a chiral one.

Apart from these two extremes, the true vertex-form for nonlocal interaction would mostly be of mixture form like $a \gamma^{\mu}+b \gamma^{\mu} \gamma_{5}$ after carrying finite steps of scaling transformation. The physics picture could be understood as follows (Figure 1). Initially, the pure vector-form $\gamma^{\mu}$ plays a rough role in describing the interaction between a point particle and an extended particle. As for the extended particle, while the interaction is very weak i.e. the interaction energy is very low, obviously it looks approximately like a point particle, i.e. not a physical particle. So $\gamma^{\mu}$ marks initially the rough interaction between two point-particles. Now let's zoom in, i.e. improving the energy (momentum) of interaction, then we can imagine that the extended particle becomes gradually sizable in contrast to original point-like. "Zooming in” is equivalent to, as we propose here, many steps of scaling transformation. After finite steps of transformation, the initial vertex $\gamma^{\mu}$ would somehow evolve to a mixture form $a \gamma^{\mu}+b \gamma^{\mu} \gamma_{5}$, with which one can use local vertex-form and form factor to interpret nonlocal interaction on certain energy scale. And the additional coefficient $a$ is assumed to be part of the form factor $F\left(q^{2}\right)$ of vector interaction, thus equivalent to the running of coupling constant. This picture coincides with that of renormalization. The conclusion of the above paragraphes also tells that while the initial interaction between points being $\gamma^{\mu} \pm \gamma^{\mu} \gamma_{5}$, then while we zooming in, the interaction between the point and the true extended particle would not change. The extreme form $\gamma^{\mu} \pm \gamma^{\mu} \gamma_{5}$ between points are the particular cases that seldom occur. The weak interaction between neutrinos and leptons belongs to such category.

\section{The Conservation Law for the Scale-Invariant Interaction}

$$
A_{\mu}^{\prime}(x, y) \bar{\psi}(y) \gamma^{\mu}\left(1 \pm \gamma_{5}\right) \psi(y)
$$

The necessity of studying the conservation law for the extreme vertices is that such vertices might exist for a very short moment in some scattering processes. According to Equation (38), to repeatedly perform the transformation succeedingly until $|N u|$ becomes very large, the incident particle would approach to a very high energy (or a very low energy) and its wave shrinks (inflates) to a very small scale (a very large scale). At such very high (low) energy scale, it is hard for the particles to shrink (inflate) more, and its interaction vertex gets to the form $A_{\mu}(x, y) \bar{\psi}(y) \gamma^{\mu}\left(1 \pm \gamma_{5}\right) \psi(y)$. This interaction vertex may appear to systems of two hadrons colliding at a very high (low) energy and exist just for a very short instant of time, though not matching any true fundamental interactions. $\gamma^{\mu}\left(1 \pm \gamma_{5}\right)$ make their sense relative to their original form $\gamma^{\mu}$ - they have evolved

Point particle

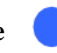

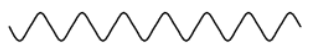

$\gamma_{\mu}$

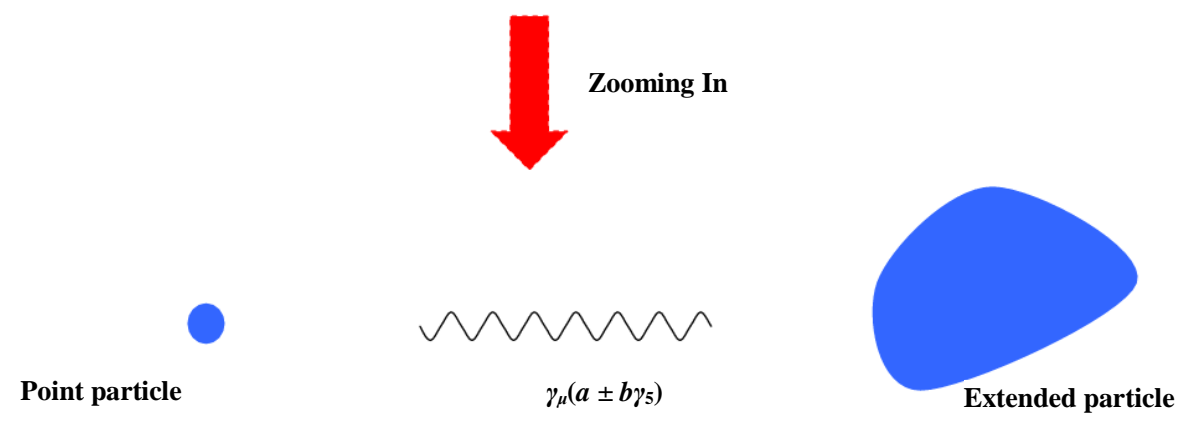

Point particle

(1) 
from the vertex-form $\gamma^{\mu} \cdot \gamma^{\mu}\left(1 \pm \gamma_{5}\right)$ describes the nonlocal interaction between a point particle and an extended particle, whereas $\gamma^{\mu}$ underlies the local interaction between the point particle and a point in extended hadron. The deviation of the vertex-form $\gamma^{\mu}\left(1 \pm \gamma_{5}\right)$ from vector $\gamma^{\mu}$ suggests that a new conservative current may appear [70]. In what follows we will study what might be the conservation law for the angular momentum of such a nonlocal system at its extreme state, as well as the impact of such conservation law on scattering processes between extended particles.

From a classical point of view, while a "soft" body (with definite mass $m$ ) rotating, its shrinkage or inflation (like zooming in or out) would not alter its total orbital angular momentum. However for a quantum particle, its shrinkage or inflation occurs only when it absorbs or releases a certain amount of energy. Such kind of energy exchange of course breaks the angular-momentum conservation by intuition. But this intuition is right only partially, since in what follows we recognize that only spin part is varied, and the spatial angular momentum is not varied due to the commutation $\left[D, M_{\mu v}\right]=0$ [61]. The case is similar to that when we extend three-dimensional rotation to four-dimensional rotation, whereby we find the 3-dimensional orbital angular momentum is not a conservative quantity any longer, unless we further include the spin angular momentum. Now with scaling transformation, we find the sum of orbital angular momentum and the spin is not conserved any longer, so we have to include the named "scalum" to find a conserved quantity. The "scalum" should be manifested by the transformation of spinors. In such sequence we call the scaling transformation an extrapolation of Poincare group, and in fact it is the very Weyl group.

A newly similar consideration of the scaling symmetry appears in Ref. [17], in which the authors discuss the scaling symmetry in 2-dimension system by using the light-cone quantum field method. And the work [44] [45] treat the scaling transformation based on a first principle form from Wilsonian method, in which some of the renormalization processes are repeated. Here we don't follow it in details of renormalization. We focus more on the application of scaling feature of renormalization to nonlocal interaction, and also on what we can infer based on such application. Earlier before there had been other efforts to associate scaling transformation to quantum field theory. None of them is satisfactory since, no perfect quantum system is found so that the corresponding Lagrangian is scaling invariant unless, the mass of involved particles are null and, the trace of energy-momentum of the system becomes zero [55]-[60]. I think a reason is that they didn't consider the spatial representation and spinor representation simultaneously. For the same reason in what follows we derive a conservation law different from those in literature.

While discussing conservation law, in Lagrangian there are at least two other additional terms to be involved, namely the kinetic term $\bar{\psi} \gamma^{\mu} p_{\mu} \psi$ and mass term $m \bar{\psi}(y) \psi(y)$. As for the kinetic term of an extended particle in the extreme condition, the momentum become light-cone like and the kinetic mass tends to zero since,

$$
m_{\text {kinetic }}^{2}=\left(\Gamma_{\mu} p^{\mu}\right)\left(\Gamma_{v} p^{v}\right)=\gamma_{\mu}\left(1-\gamma_{5}\right) p^{\mu} \gamma_{v}\left(1-\gamma_{5}\right) p^{v}=p^{2}\left(1+\gamma_{5}\right)\left(1-\gamma_{5}\right)=0,
$$

here the $m^{2}=0$ may just have comparable meaning while its momentum is very large and its mass can be ignored according to physics. For consistency we prefer to view the kinetic term as the form $\bar{\psi} \Gamma^{\mu} p_{\mu} \psi$ and now we know it keeps invariant under scaling transformation. The invariance of net mass term is ensured by the following relation if we prefer not to omit it,

$$
m \bar{\psi}(y) \psi(y)=m \bar{\psi}(y) S^{\prime-1} S^{\prime} \psi(y)=m \bar{\psi}^{\prime}(y) \psi^{\prime}(y) .
$$

In summary the Lagrangian without mass term yields

$$
L=\bar{\psi} \Gamma^{\mu} p_{\mu} \psi-g F\left(q^{2}\right) A_{\mu}\left(q^{2}\right) j^{\mu}\left(p, p^{\prime}\right) .
$$

where $j^{\mu}\left(p, p^{\prime}\right)=\bar{\psi}(p) \Gamma^{\mu} \psi\left(p^{\prime}\right)$, as the obtained vertex in the last section.

Here we mainly investigate the conserved angular momentum for Equation (41) under the transformation set $\left\{\left\{\mathrm{e}^{\frac{u}{2} \gamma^{\mu} \gamma^{\nu}}\right\}, \mathrm{e}^{\frac{u}{2} \gamma_{5}}\right\}$. First let's recall the customarily conserved quantities (Equation (42) to Equation (48)) under the usual spatial transformation, i.e. the translations and rotations. These 4-dimensional spatial transformations with infinitesimal forms are

$$
x_{\alpha} \rightarrow x_{\alpha}^{\prime}=x_{\alpha}+\delta x_{\alpha}=x_{\alpha}+\varepsilon_{\alpha \beta} x^{\beta}+\delta_{\alpha},
$$


where $\delta_{\alpha}$ is an infinitesimal displacement and $\varepsilon_{\alpha \beta}$ is an infinitesimal antisymmetric tensor for rotation in 4-dimension, $\varepsilon_{\alpha \beta}=-\varepsilon_{\beta \alpha}$. This transformation guarantees the invariance of $x_{\alpha} x^{\alpha}$ while $\delta_{\alpha}=0$. The above spatial transformation corresponds to the transformation for quantum fields as

$$
\psi_{r}(x) \rightarrow \psi_{r}^{\prime}\left(x^{\prime}\right)=\psi_{r}(x)+\frac{1}{2} \varepsilon_{\alpha \beta} S_{r s}^{\alpha \beta} \psi_{s}(x),
$$

in which the matrices elements $S_{r s}^{\alpha \beta}$ are from the spinor representation of Lorentz group [69], and in the second term both of the repeated indices stand for summations, and $\psi_{s}(x)$ 's are components in

$$
\psi^{\mathrm{T}}(x)=\left(\psi_{1}(x), \psi_{2}(x), \psi_{3}(x), \psi_{4}(x)\right) .
$$

Take $\delta_{\alpha}=0$ and impose additionally the invariance of the Lagrangian,

$$
L\left(\psi_{r}(x), \psi_{r, \alpha}(x)\right)=L\left(\psi_{r}^{\prime}\left(x^{\prime}\right), \psi_{r, \alpha}^{\prime}\left(x^{\prime}\right)\right),
$$

one gets a general conserved current (known as the Nöether current) [69] relating to angular momentum,

$$
j^{\alpha}=\frac{1}{2} \varepsilon_{\beta \gamma} \wp^{\alpha \beta \gamma},
$$

where

$$
\wp^{\alpha \beta \gamma}=\frac{\partial L}{\partial \psi_{r, \alpha}} S_{r s}^{\beta \gamma} \psi_{s}+\left[x^{\beta} \mathfrak{J}^{\alpha \gamma}-x^{\gamma} \mathfrak{J}^{\alpha \beta}\right]
$$

and

$$
\mathfrak{J}^{\alpha \beta}=\frac{\partial L}{\partial \psi_{r, \alpha}} \frac{\partial \psi_{r}(x)}{\partial x_{\beta}}-L g^{\alpha \beta} .
$$

The current Equation (45) leads to angular momentum operator in four dimensions by

$$
M^{\alpha \beta}=\int \mathbf{d}^{3} \mathbf{x} \wp^{0 \alpha \beta}=\int \mathbf{d}^{3} \mathbf{x}\left\{\left[x^{\alpha} \mathfrak{I}^{0 \beta}-x^{\beta} \mathfrak{J}^{0 \alpha}\right]+\pi_{r}(x) S_{r s}^{\alpha \beta} \psi_{s}(x)\right\},
$$

in which $\pi_{r}(x)=\frac{\partial L}{\partial \dot{\psi}_{r}(x)}$ is a conjugate field of $\psi_{r}(x)$.

Since the orbital angular momentum is not affected by scaling transformation due to $\left[D, M_{\mu v}\right]=0$, we can only add the new ingredient $\bar{\varepsilon}_{\mu \nu} \bar{S}^{\mu v}=\frac{1}{2} \varepsilon \gamma_{5}$ (from $S^{\prime}=\mathrm{e}^{\frac{u}{2} \gamma_{5}}$ ) into the total variation of field $\psi$ in Equation (43). Thus the spinor part would vary with the change of $S_{r s}^{\beta \gamma}$, as $\varepsilon_{\beta \gamma} S_{r s}^{\beta \gamma} \rightarrow \varepsilon_{\beta \gamma} S_{r s}^{\beta \gamma}+\bar{\varepsilon}_{\beta \gamma} \bar{S}_{r s}^{\beta \gamma}$. We name the latter part "scalum". The conserved current varies correspondingly

$$
\tilde{j}^{\alpha}=\frac{1}{2} \varepsilon_{\beta \gamma} \wp^{\alpha \beta \gamma}+\frac{1}{2} \bar{\varepsilon}_{\beta \gamma} \bar{\wp}^{\alpha \beta \gamma},
$$

with

$$
\bar{\varepsilon}_{\beta \gamma} \bar{\delta}^{\alpha \beta \gamma}=\frac{\partial L}{\partial \psi_{r, \alpha}} \bar{\varepsilon}_{\mu v} \bar{S}^{\mu v} \psi_{s}=\frac{\partial L}{\partial \psi_{r, \alpha}}\left(\frac{1}{2} \varepsilon \gamma_{5}\right)_{r s} \psi_{s} .
$$

Since the part $\bar{\varepsilon}_{\mu v} \bar{S}^{\mu v}=\frac{1}{2} \varepsilon \gamma_{5}$ is symmetric, to combine it with the anti-symmetric part $\varepsilon_{\beta \gamma} S_{r s}^{\beta \gamma}$ and extract a common factor $\varepsilon_{\beta \gamma}$, we have to multiply a factor $\frac{1}{6} \varepsilon_{\beta \gamma} \varepsilon^{\beta \gamma}$ ahead of the $\bar{\varepsilon}_{\mu \nu} \bar{S}^{\mu \nu}$. Then Equation (49) yields 


$$
\tilde{j}^{\alpha}=\frac{1}{2} \varepsilon_{\beta \gamma} \S^{\alpha \beta \gamma}+\frac{1}{24} \varepsilon_{\beta \gamma} \varepsilon^{\beta \gamma} \frac{\partial L}{\partial \psi_{r, \alpha}}\left(\varepsilon \gamma_{5}\right)_{r s} \psi_{s},
$$

and we should caution that in the second term only the product of the constants $\varepsilon_{\beta \gamma}$ and $\varepsilon$ is equivalent to infinitesimal constant $\varepsilon_{\beta \gamma}$ in the first term, in despite of that they have the same denotations. The left constant $\varepsilon^{\beta \gamma}$ is a normal antisymmetric constant satisfying

$$
\varepsilon^{\beta \gamma}=\left\{\begin{array}{ll}
1 & \text { if } \beta, \gamma \text { are different } \\
0 & \text { if } \beta, \gamma \text { are the same }
\end{array},\right.
$$

Thus apart from the infinitesimal parameter $\varepsilon_{\beta \gamma}$, the remaining tensor similar to Equation (46) becomes

$$
\tilde{\wp}^{\alpha \beta \gamma}=\frac{\partial L}{\partial \psi_{r, \alpha}}\left(S_{r s}^{\beta \gamma}+\frac{1}{12} w \varepsilon^{\beta \gamma}\left(\gamma_{5}\right)_{r s}\right) \psi_{s}+\left[x^{\beta} \mathfrak{J}^{\alpha \gamma}-x^{\gamma} \mathfrak{J}^{\alpha \beta}\right],
$$

the constant $w$ is responsible for the quotient between the two constants $\varepsilon_{\beta \gamma}$ and $\varepsilon$, which is assumed to be adjustable.

Now it is evident that the angular momentum Equation (46) varies correspondingly with the transformation. In this sense we conclude that the nonlocal interaction entails the new internal freedom and becomes the particle intrinsic local property. Meanwhile it brings about the extrapolation of conventional spin angular-momentum. To put it in other words, the particles with shape and those point-like seem to follow different conservation laws. For the extensive particles it is necessary to involve this correction term $\frac{\partial L}{\partial \psi_{r, \alpha}} \frac{1}{12} w \varepsilon^{\beta \gamma}\left(\gamma_{5}\right)_{r s}$ in spin part [71]. Thus when an extended particle (like proton) is smashed we shall not evaluate the polarizations of its initial state and its final state (smashed shreds of proton) in conventional way, since the initial state (proton) and the final states (smashed proton) all have their non-point size and thus the initial polarization might not be the sum of its final states (smashed proton) (Figure 2). Regarding this different conservation law may help us alleviate the spin crisis appearing in the polarized electron-nucleon scattering experiment [72]-[78].
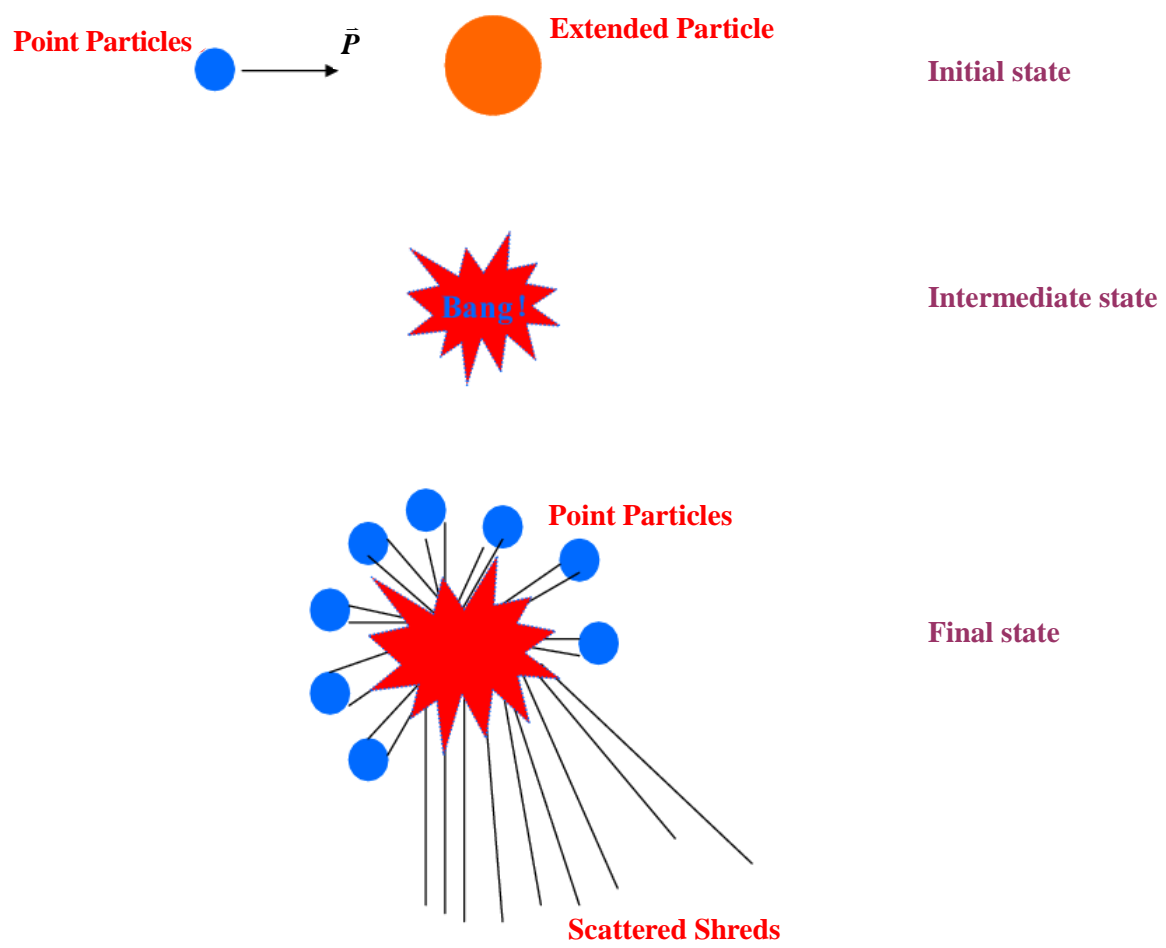

Final state

Figure 2. Schematic diagram for the colliding process between a point particle and an extended particle. 
It has been a long-standing puzzle that how the nucleon spin originates from its constituent parts, namely, the angular momentum of quarks and gluons. The conflict arose from the estimation of the total spin of proton based on the experimental value of the antisymmetric structure function $g_{1}$ [79]-[81]. The total spin $\Sigma$ (defined to be $\Sigma=\Delta u+\Delta d+\Delta s, \Delta u$ is the fraction of $u$ quark in proton's spin, and the same sense to $\Delta d$ and $\Delta s$ ) of proton relates to the structure function $g_{1}$ by generalizing Bjorken’s sum rule [82], namely,

$$
\int_{0}^{1} g_{1}^{p}\left(x, Q^{2}\right)=\frac{1}{12}\left[g_{A}^{(3)}+\frac{1}{3} g_{A}^{(8)}\right]+\frac{1}{9} \Sigma,
$$

where $g_{A}^{(3)}=\Delta u-\Delta d, \quad g_{A}^{(8)}=\Delta u+\Delta d-2 \Delta s$ are separately the iso-vector, $S U(3)$ octet. $g_{A}^{(3)}, g_{A}^{(8)}$ have been very well determined respectively from neutron $\beta$-decay and semi-leptonic hyperon decay [83]. After involving the radiative correction in perturbative QCD, the above relation can be precisely interpreted as [84]

$$
\int_{0}^{1} g_{1}^{p}\left(x, Q^{2}\right)=\frac{c_{1}\left(Q^{2}\right)}{12}\left[g_{A}^{(3)}+\frac{1}{3} g_{A}^{(8)}\right]+\frac{c_{2}\left(Q^{2}\right)}{9} \Sigma,
$$

where the coefficients $c_{1}\left(Q^{2}\right)$ and $c_{2}\left(Q^{2}\right)$ come from QCD perturbative corrections. With above knowledge, hitherto it has been well known that the value of $\Sigma$ only amounts to 1/3 of total proton's spin.

People once conceived gluons' spin may contribute much to proton's spin, but recent experimental analysis [85] supports merely small fraction of gluon's contribution. Another recent flurry has been the focus on the decomposition of angular momentum of quark and gluon into spin part and orbital angular momentum part based on the gauge-invariant QCD dynamics [77] [83] [86]-[91]. But the ways to treat angular momentum of bounded quarks are so controversial that hitherto there has been no widely accepted scheme. In a very recent paper, Ji et al. [75] refined the sum rule using generalized parton distribution (GPD) method, which may improve further the evaluation of $\Sigma$.

Now let's focus on the coefficients of $c_{1}\left(Q^{2}\right)$ and $c_{2}\left(Q^{2}\right)$ in Equation (55), whose accurate values are based on the perturbative calculation in QCD. For instance, the coefficients $c_{1}\left(Q^{2}\right)$ reads [82]

$$
c_{1}\left(Q^{2}\right)=1-\left(\frac{\alpha_{s}}{\pi}\right)-3.58333\left(\frac{\alpha_{s}}{\pi}\right)^{2}+\cdots,
$$

in which $\alpha_{s}\left(Q^{2}\right)$ may have a running value with respect to $Q^{2}$, roughly around $0.1 \backslash$ symbol $\{126\} 0.3$. Because the scale transformation is somehow derived from the renormalization group, including the running of charges etc., one may be aware of that the corrections in Equation (56) are to some extent equal to the scaling transformation. And the corrections from Equation (56) might also be consistent with effect that interpreted by Equation (53). Though the corrections have not made the coefficients $c_{1}\left(Q^{2}\right)$ and $c_{2}\left(Q^{2}\right)$ deviate so much from 1 , we know the corrections actually affect the value of $\Sigma$. When the scale approaches to the nonperturbative regime and $\alpha_{s}\left(Q^{2}\right)$ becomes larger, the expression of Equation (56) however, may lose its validation. Whereas we note our scaling transformation happens to be responsible for the shift between the perturbative and nonperturbative regimes since the dilation (shrinkage) occurs accompanying with the loss (injection) of energy. $\mathrm{Nu} \rightarrow \pm \infty$ may imply the coupling constant $\alpha_{s} \rightarrow \infty$ according to our previous understanding of the form factor, so the extreme states are really relevant. We thus speculate that the scaling transformation might be helpful to transform the spin value from perturbative scale to nonperturbative scale, or vice versa. In this sense the transformation method could be a way to find an explanation on the spin crisis of proton. And further investigation is in progress. While the $S U(3)$ group steps in, some unexpected effects may occur.

\section{The Impact of Vertex $F\left(q^{2}\right) A_{\mu}(k) \bar{\psi}(p) \gamma^{\mu}\left(a+b \gamma_{5}\right) \psi(p)$ on Polarized Scattering}

In this section we will discuss that after finite steps of scaling transformations, what is the contribution of the evolving vertex $A_{u}(k) \bar{\psi}(p) \gamma^{\mu}\left(a+b \gamma_{5}\right) \psi(p)$ to the scattering processes. Analogous to the inelastic e-p scattering, where the assumed vertex $A \gamma^{\mu}+B \sigma^{\mu v} p_{v}$ yields some observed structures $W^{\mu v}$ in cross-section [92], here we are concerned about what the structures the evolving vertex-form $\gamma^{\mu}\left(a+b \gamma_{5}\right)$ would lead to. Although we work following the analogy, we should caution that we focus on elastic scattering, rather than inelastic scattering. At the end of this section we arrive at the conclusions that the part $b \gamma^{\mu} \gamma_{5}$ contributes nothing to 
the normal unpolarized cross-section of elastic scattering, so effectively it doesn't change the conventional structure form. However, for the polarized scattering, there appears exceptional terms additional to the original structure function.

Firstly, let's carry out the structure function of unpolarized cross-section by averaging over the initial spins and summing over the final spins [92]. Without losing generality, let's suppose that it is the very case for an electron (a point fermion) incident on nucleon (an extended fermion) (Figure 3). By conventional steps, one finds that the evolving vertex-form $\gamma^{\mu}\left(a+b \gamma_{5}\right)$ yields the following scattering tensor,

$$
W_{\mu \nu}^{(p)}=\frac{1}{2} \operatorname{Tr}\left[\frac{q_{2}+M}{2 M} \gamma_{\mu}\left(a+b \gamma_{5}\right) \frac{p_{2}+M}{2 M} \gamma_{v}\left(a+b \gamma_{5}\right)\right]
$$

and apart from the coefficient $\frac{1}{2}$, the trace can be separated into four terms

$$
\begin{aligned}
W_{\mu \nu}^{(p)}= & W_{\mu \nu}^{(1)}\left(a^{2}\right)+W_{\mu \nu}^{(2)}(a b)+W_{\mu \nu}^{(3)}(b a)+W_{\mu \nu}^{(4)}\left(b^{2}\right) \\
= & \frac{1}{2} a^{2} \operatorname{Tr}\left[\frac{q_{2}+M}{2 M} \gamma_{\mu} \frac{p_{2}+M}{2 M} \gamma_{\nu}\right]+\frac{1}{2} a b \operatorname{Tr}\left[\frac{q_{2}+M}{2 M} \gamma_{\mu} \frac{p_{2}+M}{2 M} \gamma_{\nu} \gamma_{5}\right] \\
& +\frac{1}{2} b a \operatorname{Tr}\left[\frac{q_{2}+M}{2 M} \gamma_{\mu} \gamma_{5} \frac{p_{2}+M}{2 M} \gamma_{\nu}\right]+\frac{1}{2} b^{2} \operatorname{Tr}\left[\frac{q_{2}+M}{2 M} \gamma_{\mu} \gamma_{5} \frac{p_{2}+M}{2 M} \gamma_{\nu} \gamma_{5}\right],
\end{aligned}
$$

and the result of the first term is well-known, it is

$$
W_{\mu \nu}^{(1)}\left(a^{2}\right)=\frac{a^{2}}{2 M^{2}}\left[q_{2 \mu} p_{2 v}+q_{2 v} p_{2 \mu}-\left(q_{2} \cdot p_{2}-M^{2}\right) g_{\mu v}\right]
$$

the second term is

$$
W_{\mu \nu}^{(2)}(a b)=\frac{a b}{M^{2}} i \varepsilon_{\mu \nu \rho \sigma} q_{2}^{\rho} p_{2}^{\sigma}
$$

and the third term results in the same

$$
W_{\mu \nu}^{(3)}(b a)=\frac{a b}{M^{2}} i \varepsilon_{\mu \nu \rho \sigma} q_{2}^{\rho} p_{2}^{\sigma} .
$$

The last term has the same form as the first term, $W_{\mu \nu}^{(1)}\left(a^{2}\right)$, apart from the coefficient

$$
W_{\mu \nu}^{(4)}\left(b^{2}\right)=\frac{b^{2}}{2 M^{2}}\left[q_{2 \mu} p_{2 v}+q_{2 \nu} p_{2 \mu}-\left(q_{2} \cdot p_{2}-M^{2}\right) g_{\mu \nu}\right] \text {. }
$$

Figure 3. The Feynman graph for calculating the scattering cross-section for point particles, with the vertexform $A_{\mu} \gamma^{\mu}\left(a+b \gamma^{5}\right)$. 
We note the new structure functions are from Equations (60), (61), whose contribution however, would vanish since the tensor $\varepsilon_{\mu \nu \rho \sigma}$ are antisymmetric, while the tensor of lepton part $L^{\mu \nu}$ coupled to them is symmetric with respect to indices $\mu, v$.

Secondly, let's consider the polarized cross-section. Now we do not fix the initial or the final spin states and leave the spin operator in the potential. With the same marks as in Figure 3 and without the propagator, we write the polarized amplitude (potential) by using the Dirac spinors as follows

$$
\begin{aligned}
M_{A V}(\boldsymbol{p}, \boldsymbol{q}, \boldsymbol{k})= & \bar{\psi}_{1} \gamma_{1 \mu}\left(a+b \gamma_{5}\right) \psi_{1} \bar{\psi}_{2} \gamma_{2}^{\mu}\left(a+b \gamma_{5}\right) \psi_{2} \\
= & a^{2} \bar{\psi}_{1} \gamma_{1 \mu} \psi_{1} \bar{\psi}_{2} \gamma_{2}^{\mu} \psi_{2}+a b \bar{\psi}_{1} \gamma_{1 \mu} \psi_{1} \bar{\psi}_{2} \gamma_{2}^{\mu} \gamma_{5} \psi_{2} \\
& +b a \bar{\psi}_{1} \gamma_{1 \mu} \gamma_{5} \psi_{1} \bar{\psi}_{2} \gamma_{2}^{\mu} \psi_{2}+b^{2} \bar{\psi}_{1} \gamma_{1 \mu} \gamma_{5} \psi_{1} \bar{\psi}_{2} \gamma_{2}^{\mu} \gamma_{5} \psi_{2},
\end{aligned}
$$

Only the terms with coefficients $a b$ and $b a$ (henceforth we denote the two terms as $\{a b\}$ and $\{b a\}$ ) are new, and the results for the other two terms $\{a a\}$ and $\{b b\}$ can be found in Ref. [93]. The term $\{a b\}$ can be tidied up into

$$
\begin{aligned}
M_{\{a b\}} & =\bar{U}\left(\boldsymbol{p}_{1}\right) \gamma_{1 \mu} U\left(\boldsymbol{q}_{1}\right) \bar{U}\left(\boldsymbol{p}_{2}\right) \gamma_{2}^{\mu} \gamma_{5} U\left(\boldsymbol{q}_{2}\right) \\
& =\bar{U}\left(\boldsymbol{p}_{1}\right) \gamma_{10} U\left(\boldsymbol{q}_{1}\right) \bar{U}\left(\boldsymbol{p}_{2}\right) \gamma_{2}^{0} \gamma_{5} U\left(\boldsymbol{q}_{2}\right)-\bar{U}\left(\boldsymbol{p}_{1}\right) \gamma_{1} U\left(\boldsymbol{q}_{1}\right) \cdot \bar{U}\left(\boldsymbol{p}_{2}\right) \gamma_{2} \gamma_{5} U\left(\boldsymbol{q}_{2}\right),
\end{aligned}
$$

where the indices 1, 2 represent respectively the first and the second particles. Substitute the concrete form of Dirac spinor $U(\boldsymbol{p})=\sqrt{\frac{E+m}{2 E}}\left(\begin{array}{c}1 \\ \frac{\boldsymbol{\sigma} \cdot \boldsymbol{p}}{E+m}\end{array}\right)$ and $\bar{U}(\boldsymbol{p})=U^{\dagger}(\boldsymbol{p}) \gamma_{0} \quad$ (where $E=\sqrt{\boldsymbol{p}^{2}+m^{2}}$ ) into the above equation, and after lengthy calculation, it yields

$$
\begin{aligned}
& M_{\{a b\}}=A\left\{\frac{\boldsymbol{\sigma}_{2} \cdot \boldsymbol{p}_{2}}{E\left(\boldsymbol{p}_{2}\right)+m_{2}}+\frac{\boldsymbol{\sigma}_{2} \cdot \boldsymbol{q}_{2}}{E\left(\boldsymbol{q}_{2}\right)+m_{2}}+\frac{\boldsymbol{\sigma}_{1} \cdot \boldsymbol{p}_{1}}{E\left(\boldsymbol{p}_{1}\right)+m_{1}} \frac{\boldsymbol{\sigma}_{1} \cdot \boldsymbol{q}_{1}}{E\left(\boldsymbol{q}_{1}\right)+m_{1}} \frac{\boldsymbol{\sigma}_{2} \cdot \boldsymbol{p}_{2}}{E\left(\boldsymbol{p}_{2}\right)+m_{2}}\right. \\
& +\frac{\boldsymbol{\sigma}_{1} \cdot \boldsymbol{p}_{1}}{E\left(\boldsymbol{p}_{1}\right)+m_{1}} \frac{\boldsymbol{\sigma}_{1} \cdot \boldsymbol{q}_{1}}{E\left(\boldsymbol{q}_{1}\right)+m_{1}} \frac{\boldsymbol{\sigma}_{2} \cdot \boldsymbol{q}_{2}}{E\left(\boldsymbol{q}_{2}\right)+m_{2}}-\left[\frac{\boldsymbol{\sigma}_{1} \cdot \boldsymbol{p}_{1}}{E\left(\boldsymbol{p}_{1}\right)+m_{1}} \underline{\boldsymbol{\sigma}}_{1} \frac{\boldsymbol{\sigma}_{2} \cdot \boldsymbol{p}_{2}}{E\left(\boldsymbol{p}_{2}\right)+m_{2}} \underline{\boldsymbol{\sigma}}_{2} \frac{\boldsymbol{\sigma}_{2} \cdot \boldsymbol{q}_{2}}{E\left(\boldsymbol{q}_{2}\right)+m_{2}}\right. \\
& \left.\left.+\frac{\boldsymbol{\sigma}_{1} \cdot \boldsymbol{p}_{1}}{E\left(\boldsymbol{p}_{1}\right)+m_{1}} \boldsymbol{\sigma}_{1} \cdot \boldsymbol{\sigma}_{2}+\underline{\boldsymbol{\sigma}}_{1} \frac{\boldsymbol{\sigma}_{1} \cdot \boldsymbol{q}_{1}}{E\left(\boldsymbol{q}_{1}\right)+m_{1}} \underline{\boldsymbol{\sigma}}_{2}+\underline{\boldsymbol{\sigma}}_{1} \frac{\boldsymbol{\sigma}_{1} \cdot \boldsymbol{q}_{1}}{E\left(\boldsymbol{q}_{1}\right)+m_{1}} \frac{\boldsymbol{\sigma}_{2} \cdot \boldsymbol{p}_{2}}{E\left(\boldsymbol{p}_{2}\right)+m_{2}} \underline{\boldsymbol{\sigma}}_{2} \frac{\boldsymbol{\sigma}_{2} \cdot \boldsymbol{q}_{2}}{E\left(\boldsymbol{q}_{2}\right)+m_{2}}\right]\right\},
\end{aligned}
$$

here we use underlines to denote the inner product and

$$
A=\sqrt{\frac{E\left(\boldsymbol{p}_{1}\right)+m_{1}}{2 E\left(\boldsymbol{p}_{1}\right)} \frac{E\left(\boldsymbol{p}_{2}\right)+m_{2}}{2 E\left(\boldsymbol{p}_{2}\right)} \frac{E\left(\boldsymbol{q}_{1}\right)+m_{1}}{2 E\left(\boldsymbol{q}_{1}\right)} \frac{E\left(\boldsymbol{q}_{2}\right)+m_{2}}{2 E\left(\boldsymbol{q}_{2}\right)}} \approx 1-\frac{1}{8 m_{1}^{2}}\left(\boldsymbol{p}_{1}^{2}+\boldsymbol{q}_{1}^{2}\right)-\frac{1}{8 m_{2}^{2}}\left(\boldsymbol{p}_{2}^{2}+\boldsymbol{q}_{2}^{2}\right) .
$$

The second step follows while using the approximation $E\left(\boldsymbol{p}_{1}\right) \approx m_{1}+\frac{\boldsymbol{p}_{1}^{2}}{2 m_{1}}$ and to the order of $\frac{\boldsymbol{p}^{2}}{m^{2}}$. With this approximation, one further gets

$$
\begin{aligned}
M_{\{a b\}} & =A\left\{\frac{1}{2 m_{2}} \boldsymbol{\sigma}_{2} \cdot\left(\boldsymbol{p}_{2}+\boldsymbol{q}_{2}\right)-\frac{1}{2 m_{1}}\left[\boldsymbol{\sigma}_{1} \cdot \boldsymbol{p}_{1} \boldsymbol{\sigma}_{1} \cdot \boldsymbol{\sigma}_{2}-\underline{\sigma}_{1} \boldsymbol{\sigma}_{1} \cdot \boldsymbol{q}_{1} \underline{\sigma}_{2}\right]\right\} \\
& =A\left\{\frac{1}{2 m_{2}} \boldsymbol{\sigma}_{2} \cdot\left(\boldsymbol{p}_{2}+\boldsymbol{q}_{2}\right)-\frac{1}{2 m_{1}}\left[\left(\boldsymbol{p}_{1}-\boldsymbol{q}_{1}\right) \cdot \boldsymbol{\sigma}_{2}-i\left(\boldsymbol{\sigma}_{1} \times \boldsymbol{\sigma}_{2}\right) \cdot\left(\boldsymbol{p}_{1}+\boldsymbol{q}_{1}\right)\right]\right\},
\end{aligned}
$$

in the second step of the above equation we have used the following relations

$$
\sigma_{i} \sigma_{j}=\delta_{i j}+i \varepsilon_{i j k} \sigma_{k} \text {, }
$$

and 


$$
(\boldsymbol{\sigma} \cdot \boldsymbol{A})(\boldsymbol{\sigma} \cdot \boldsymbol{B})=A_{i} B_{j} \sigma_{i} \sigma_{j}=\boldsymbol{A} \cdot \boldsymbol{B}+i(\boldsymbol{A} \times \boldsymbol{B}) \cdot \boldsymbol{\sigma} .
$$

Likewise, we obtain $M_{\{b a\}}$ as follows

$$
M_{\{b a\}} \simeq A\left\{\frac{1}{2 m_{1}} \boldsymbol{\sigma}_{1} \cdot\left(\boldsymbol{p}_{1}+\boldsymbol{q}_{1}\right)-\frac{1}{2 m_{2}}\left[\left(\boldsymbol{p}_{2}+\boldsymbol{q}_{2}\right) \cdot \boldsymbol{\sigma}_{1}+i\left(\boldsymbol{\sigma}_{1} \times \boldsymbol{\sigma}_{2}\right) \cdot\left(\boldsymbol{p}_{2}-\boldsymbol{q}_{2}\right)\right]\right\} .
$$

We note that the terms $M_{\{a b\}}$ and $M_{\{b a\}}$ do not appear in those cross-sections derived from any single of five Lorentz-invariant currents $\bar{\psi}(p) \psi(p), \bar{\psi}(p) \gamma^{\mu} \psi(p), \bar{\psi}(p) \gamma_{5} \psi(p), \bar{\psi}(p) \gamma_{5} \gamma^{\mu} \psi(p), \bar{\psi}(p) \gamma^{\mu} \gamma^{v} \psi(p)$, unless some of them are mixed. Thus we realize that $M_{\{a b\}}$ and $M_{\{b a\}}$ can actually occur if the current is the weak current $\bar{\psi}(p) \gamma^{\mu}\left(1-\gamma_{5}\right) \psi(p)$, for instance in the scattering of neutrino incident on electron. However, since the intermediate $\mathrm{Z}$ boson is very heavy and thus the scattering involving weak interaction only appears in very high energy, we may avoid the case by testing effects of nonlocality in somewhat lower energy regime. Moreover, mostly the vertex we meet in nonlocal current must be $\bar{\psi}(p) \gamma^{\mu}\left(a+b \gamma_{5}\right) \psi(p)$ instead of pure extremes $\bar{\psi}(p) \gamma^{\mu}\left(1 \pm \gamma_{5}\right) \psi(p)$, which leave coefficients $a, b$ to adjust. Unexpectedly, if the nonlocal extreme vertices were mixed or entangled with weak interaction and were evidenced by experiments, then it must be a most intriguing topic deserving further investigation.

We would like to present a simple gedanken experiment to test nonlocal effect due to the handedness terms, which are all proportional to helicity $\boldsymbol{\sigma} \cdot \boldsymbol{p}$ in one manner or another, as shown in Equations (67), (70). For the feasibility of the experiment, we turn from hadron dynamics to molecular scale to test the prediction of Equations (67), (70). Imagine that an electron scattered away from a simple atom like hydrogen, which stays in its ground state, so that no orbital angular momentum is involved in the scattering processes. Meanwhile we should control the energy of incident electron to be low enough so that other orbital states of hydrogen-atom are not involved. Maybe the energy should $E \leq 1 \mathrm{eV}$ or several $\mathrm{eVs}$ (with wavelength less than $2 \AA$ ), which is largely lower than its first threshold of transitions. The electron's energy have to be controlled precisely to limit its wavelength less than hydrogen-atom diameter and meanwhile not so short as to cause transition of hydrogen-atom. If actually the energy of the electron is not easy to control, we may directly use the scattering between ground-state hydrogen atoms instead of the scattering between electron and hydrogen-atom. In such scenario the total angular momentum of hydrogen atom is its spin, and the nucleon magnetic moment is omitted for its small fraction in the total (about 1 in 2000). And the spreading electron cloud of hydrogen atom meets the case of our nonlocal description. Such hydrogen atom could be good testing ground for nonlocal predictions.

The proposed experiment is to use polarized electrons (or hydrogen atoms) colliding on polarized hydrogenatom (polarized by magnetic field). Different from the previous calculations on polarized electron-electron (e-e) scattering [94] and e-H scattering [95]-[100], the main results there are shown in Equations (67), (70), which is characterized by terms like $i\left(\boldsymbol{\sigma}_{1} \times \boldsymbol{\sigma}_{2}\right) \cdot\left(\boldsymbol{p}_{2}-\boldsymbol{q}_{2}\right)$. Such term differs from the normal handedness term $\boldsymbol{\sigma} \cdot \boldsymbol{p}$ in that it permits the existence of two perpendicular spins $\sigma_{1}$ and $\sigma_{2}$. Whereas the previous test-experiments on spin asymmetry [96] mainly focused on the parallel or anti-parallel difference, as

$$
A_{1 s, 1 s}(\theta)=\frac{\sigma_{\uparrow \downarrow}-\sigma_{\uparrow \uparrow}}{\sigma_{\uparrow \downarrow}+\sigma_{\uparrow \uparrow}},
$$

or

$$
A_{1 s, 1 s}(\theta)=\frac{\sigma_{\Rightarrow}-\sigma_{\Leftarrow}}{\sigma_{\Rightarrow}+\sigma_{\Leftarrow}},
$$

where $\sigma_{\uparrow \downarrow}, \sigma_{\uparrow \uparrow}$, or $\sigma_{\Rightarrow}, \sigma_{\Leftarrow}$ mean the parallel or anti-parallel in common sense. However, in our case, we suggest a new asymmetric parameter

$$
A_{1 s, 1 s}(\theta)=\frac{\sigma_{\rightarrow \uparrow}-\sigma_{\uparrow \rightarrow}}{\sigma_{\rightarrow \uparrow}+\sigma_{\uparrow \rightarrow}},
$$

which has never been investigated in previous theoretical study [95]-[100] or experiments [101]-[104]. But this term may contribute even smaller fractions to total cross-section, since the sum of all spin-dependent terms have 
attributed just minor fraction in total cross-section. The calculation details can follow the paper [94], concerning additionally the present interaction. If any experiment gets a nontrivial parameter $\frac{\sigma_{\rightarrow \uparrow}-\sigma_{\uparrow \rightarrow}}{\sigma_{\rightarrow \uparrow}+\sigma_{\uparrow \rightarrow}}$ then it proves our predictions. And maybe each of the three parameters $\left(\frac{\sigma_{\uparrow \downarrow}-\sigma_{\uparrow \uparrow}}{\sigma_{\uparrow \downarrow}+\sigma_{\uparrow \uparrow}}, \frac{\sigma_{\Rightarrow}-\sigma_{\xi}}{\sigma_{\Rightarrow}+\sigma_{\xi}}\right.$, and $\left.\frac{\sigma_{\rightarrow \uparrow}-\sigma_{\uparrow \rightarrow}}{\sigma_{\rightarrow \uparrow}+\sigma_{\uparrow \rightarrow}}\right)$ would deviate from former evaluations in literature if involving all of the handedness terms $\boldsymbol{\sigma} \cdot \boldsymbol{p}$ in Equations (67), (70).

\section{Conclusions and Discussions}

In this paper we have discussed elaborately the role of scaling transformation in nonlocal interaction. This transformation pertains to describing the relationship of different energy/space-time scales in scattering between (fermion) hadrons. The scaling transformation is recognized/constructed based on the conclusions of RGM and the popular expressions of conformal group. The most significant feature of this paper is to combine its spinor representation $\gamma_{5}$ and coordinate representation $i x_{\mu} \partial^{\mu}$ together. To this end, we surmise there is a local vertex $\Gamma^{\mu}$ transforming as $S^{\prime-1} \Gamma^{\mu} S^{\prime}=\Lambda_{v}^{\prime \mu} \Gamma^{\nu}$, in which $S^{\prime}=\mathrm{e}^{\frac{u}{2} \gamma_{5}}$, resembling Lorentz transformation acting on vector vertex, $S^{-1} \gamma^{\mu} S=\Lambda_{v}^{\mu} \gamma^{v}$, where $S$ corresponds to spinor representation of Lorentz transformation. In this way we obtain the scaling invariant vertices $\Gamma^{\mu}=\gamma^{\mu}\left(1 \pm \gamma_{5}\right)$, which means the invariance of interaction vertex $A_{\mu}(x) \bar{\psi}(x) \Gamma^{\mu} \psi(x)$ while performing the scaling transformation.

Based on the knowledge that the transformation $S^{\prime}$ is applied repeatedly to vector vertex

$$
\mathrm{e}^{-\frac{u}{2} \gamma_{5}} \gamma^{\mu} \mathrm{e}^{\frac{u}{2} \gamma_{5}} \simeq \gamma^{\mu}+u \gamma^{\mu} \gamma_{5}=(1-u) \gamma^{\mu}+u \gamma^{\mu}\left(1+\gamma_{5}\right)=(1+u) \gamma^{\mu}-u \gamma^{\mu}\left(1-\gamma_{5}\right),
$$

one finds the varying coefficients ahead of $\gamma^{\mu}$, which matches the running coupling constant occurring in RGM. As for vertices $\gamma_{\mu}\left(1 \pm \gamma_{5}\right)$, here they are viewed as extremes of normal vector vertex-form $\gamma^{\mu}$ after infinite steps of scaling transformation since, $N u \rightarrow \pm \infty$,

$$
\left(\mathrm{e}^{-\frac{u}{2} \gamma_{5}}\right)^{N} \gamma^{\mu}\left(\mathrm{e}^{\frac{u}{2} \gamma_{5}}\right)^{N}=\gamma^{\mu} \cosh N u+\gamma^{\mu} \gamma_{5} \sinh N u \rightarrow \gamma_{\mu}\left(1 \pm \gamma_{5}\right) .
$$

We also call $\gamma_{\mu}\left(1 \pm \gamma_{5}\right)$ the vertices at extreme condition, which might be the system of very high energy or at very low temperature. We further discuss the conservation law for these extreme vertices, for which an extra intrinsic-degree named scalum is introduced into the total angular momentum. Based on the experience from renormalization, the parameter $\mu$ is somewhat equivalent to such a degree of freedom. It is natural for us to associate the results of conservation law with the spin crisis of nucleons, responding to the appearance of the scalum.

The extreme states as well as the extreme vertex may not exist in nature. However, by the inquiring and inferring process we recognize that the conformal group exists more like for running properties rather than for invariance of quantum fields. For an extended particle involved in a scattering at certain energy, we have to make corresponding scaling transformations to interpret locally its interaction vertex. Assume a nonlocal interaction interpreted initially/unphysically by exchanging vector bosons, then a general interaction-vertex $\gamma^{\mu}\left(a+b \gamma_{5}\right)$ exists, with which we use local vertex-form to interpret the nonlocal interaction. The general interaction-vertex has effects on polarized scattering rather than unpolarized scattering. Accordingly we propose a gedanken experiment to test our predictions on nonlocal interaction. The experiment is based on the scattering between a charged point-particle and the ground state of a hydrogen. That is recognized as a good method to test nonlocal interaction-vertex, since the cloud of ground-state electron distributes around the nucleon so that it forms a nonlocal region [105], meanwhile all of its angular momentum is the spin of the electron.

Although the dynamics used in this paper mostly stems from the perturbative dynamics, it opens a door for our understanding to nonperturbative dynamics. There have been continuous efforts to study nonperturbative interaction ever since the birth of renormalization [106]-[108]. To apply somehow the scale parameter of renormalization to intermediate-strong-interaction was the primary goal of this paper. Furthermore an even stronger motivation is to develop an analytic non-perturbation method to understand such intermediate-strong-interaction. 
The motivation has driven us to apply transformation instead of solely the scale parameter to nonperturbative interactions. Some other nonlocal theories have made efforts to link the nonlocal interaction with renormalization, for instance in Ref. [6] [9] [31] [32]. But none of them used transformation method, which was laid there years before [109] [110]. In our results, the appearance of $\gamma_{5}$ in both the scaling transformation and the nonlocal vertex-form gives us the confidence that we might have unveiled a truth of nonperturbative dynamics. Since when a current quark gains its mass non-perturbatively to become a constituent quark, the $\gamma_{5}$ as well as the chiral-symmetry breaking would occur. Next if possible we aim to construct a general description of nonperturbative systems based on their nonlocal properties.

\section{Acknowledgements}

I am grateful to the hospitality of Prof. Y. B. Dong and Prof. P. Wang when I visited High Energy Institute of Chinese Academy, as well as the fruitful discussions with them. The Project Sponsored by the Scientific Research Foundation for the Returned Overseas Chinese Scholars, State Education Ministry and Fundamental Research Funds for the Central Universities.

\section{References}

[1] Ganor, O.J. (2007) Physical Review D, 75, Article ID: 025002. http://dx.doi.org/10.1103/PhysRevD.75.025002

[2] Minton, G. and Sahakian, V. (2008) Physical Review D, 77, Article ID: 026008. http://dx.doi.org/10.1103/PhysRevD.77.026008

[3] Fodor, Z. and Hoelbling, C. (2012) Reviews of Modern Physics, 84, 449-495. http://dx.doi.org/10.1103/RevModPhys.84.449

[4] Timofeyuk, N.K. and Johnson, R.C. (2013) Physical Review Letters, 110, Article ID: 112501. http://dx.doi.org/10.1103/PhysRevLett.110.112501

[5] Eliezer, D.A. and Woodard, R.P. (1989) Nuclear Physics B, 325, 389-469. http://dx.doi.org/10.1016/0550-3213(89)90461-6

[6] Moffat, J.W. (1989) Physical Review D, 39, 3654-3671. http://dx.doi.org/10.1103/PhysRevD.39.3654

[7] Evens, D., Moffat, J.W., Kleppe, G. and Woodard, R.P. (1991) Physical Review D, 43, 499-519. http://dx.doi.org/10.1103/PhysRevD.43.499

[8] Kleppe, G. and Woodard, R.P. (1992) Nuclear Physics B, 388, 81-112. http://dx.doi.org/10.1016/0550-3213(92)90546-N

[9] Moffat, J.W. (1990) Physical Review D, 41, 1177-1184. http://dx.doi.org/10.1103/PhysRevD.41.1177

[10] Neves, M.J., Farina, C. and Cougo-Pinto, M.V. (2010) Physical Review Letters, 105, Article ID: 211601. http://dx.doi.org/10.1103/PhysRevLett.105.211601

[11] Campo, D. (2010) arXiv:1004.5324. http://arxiv.org/abs/1004.5324

[12] Brüning, E. and Nagamachi, S. (2004) Journal of Mathematical Physics, 45, 2199. http://dx.doi.org/10.1063/1.1737055

[13] Haque, A. and Joglekar, S.D. (2009) Journal of Physics A: Mathematical and Theoretical, 42, Article ID: 065401. http://dx.doi.org/10.1088/1751-8113/42/6/065401

[14] Dowker, J.S. (2009) arXiv:0902.4180. http://arxiv.org/abs/0902.4180

[15] Barnaby, N. (2011) Nuclear Physics B, 845, 1-29. http://dx.doi.org/10.1016/j.nuclphysb.2010.11.016

[16] Calcagni, G., Montobbio, M. and Nardelli, G. (2008) Physics Letters B, 662, 285-289. http://dx.doi.org/10.1016/j.physletb.2008.03.024

[17] Hofman, D.M. and Strominger, A. (2011) Physical Review Letters, 107, Article ID: 161601. http://dx.doi.org/10.1103/PhysRevLett.107.161601

[18] Moffat, J.W. (2011) arXiv:1104.5706. http://arxiv.org/abs/1104.5706

[19] Zhou, L.-J., Wu, Q. and Ma, W.-X. (2008) Communications in Theoretical Physics, 50, 161-174. http://dx.doi.org/10.1088/0253-6102/50/1/33

[20] Pimikov, A.V., Bakulev, A.P. and Stefanis, N.G. (2010) Nuclear Physics B-Proceedings Supplements, 198, $182-185$. Also at arXiv:0909.4624v1 [hep-ph].

[21] Wataghin, G. (1934) Zeitschrift für Physik, 88, 92-98. http://dx.doi.org/10.1007/BF01352311 
[22] Yukawa, H. (1950) Physical Review, 80, 1047-1052. (ibid, 77, 849 (1950); ibid 76, 300 (1949))

[23] Yennie, D.R. (1950) Physical Review, 80, 1053-1061. http://dx.doi.org/10.1103/PhysRev.80.1053

[24] Kristensen, P., Moller, C. and Dan, K. (1952) Vidensk. Selsk. Mat.-Fys. Medd., 27.

[25] Bloch, C. and Dan, K. (1952) Vidensk. Selsk. Mat.-Fys. Medd., 27.

[26] Pauli, W. (1953) Il Nuovo Cimento, 10, 648-667. http://dx.doi.org/10.1007/BF02815288

[27] Hayashi, C. (1953) Progress of Theoretical Physics, 10, 533-548. ibid, 11, 226 (1954).

[28] Lee, T.D. and Yang, C.N. (1957) Physical Review, 108, 1611-1614. http://dx.doi.org/10.1103/PhysRev.108.1611

[29] Dirac, P.A.M. (1962) Proceedings of the Royal Society A, 268, 57-67. http://dx.doi.org/10.1098/rspa.1962.0124

[30] Kirzhnits, D.A. (1967) Soviet Physics Uspekhi, 9, 692-700. Translated from Uspekhi Fizicheskikh Nauk, 90, $129-142$. (Sep. 1966).

[31] Efimov, G.V. (1967) Communications in Mathematical Physics, 5, 42-56. http://dx.doi.org/10.1007/BF01646357

[32] Efimov, G.V. (1972) Annals of Physics, 71, 466-485. http://dx.doi.org/10.1016/0003-4916(72)90127-3

[33] Alebastrov, V.A. and Efimov, G.V. (1973) Communications in Mathematical Physics, 31, 1-24. http://dx.doi.org/10.1007/BF01645588

[34] Alebastrov, V.A., Efimov, G.V. and Seltzer, S.Z. (1973) Annals of Physics, 76, 251-280. http://dx.doi.org/10.1016/0003-4916(73)90450-8

[35] Bloch, C. and Dan, K. (1950) Vidensk. Selsk. Mat.-Fys. Medd, 26.

[36] Marnelius, R. (1974) Physical Review D, 10, 3411. (Equation (2.3) and Equation (2.8); ibid, 8, 2472 (1973))

[37] Yang, C.N. and Feldman, D. (1950) Physical Review, 79, 972-978. http://dx.doi.org/10.1103/PhysRev.79.972

[38] Kleppe, G. and Woodard, R.P. (1992) Nuclear Physics B, 388, 81-112. http://dx.doi.org/10.1016/0550-3213(92)90546-N

[39] Clayton, M.A. (2001) Modern Physics Letters A, 16, 1117-1127. http://dx.doi.org/10.1142/S0217732301004327

[40] Calian, V. and Stoenescu, G. (2000) arXiv:hep-th/0011163. http://arxiv.org/abs/hep-th/0011163

[41] Raje Bhageerathi, P.C. and Eapen, K. (1998) International Journal of Modern Physics A, 13, 797-829. http://dx.doi.org/10.1142/S0217751X98000354

[42] Kleppe, G. and Woodard, R.P. (1993) Annals of Physics, 221, 106-164. http://dx.doi.org/10.1006/aphy.1993.1006

[43] Wang, P. (2011) Chinese Physics C, 35, 223-227. http://dx.doi.org/10.1088/1674-1137/35/3/003

[44] Cubero, A.C. and Orland, P. (2011) Physical Review D, 84, Article ID: 065034. http://dx.doi.org/10.1103/PhysRevD.84.065034

[45] Orland, P. and Xiao, J. (2009) Physical Review D, 80, Article ID: 016005. http://dx.doi.org/10.1103/PhysRevD.80.016005

[46] Su, J.-C., Yi, X.-X. and Cao, Y.-H. (1999) Journal of Physics G: Nuclear and Particle Physics, 25, 2325-2344. http://dx.doi.org/10.1088/0954-3899/25/12/303

[47] Su, J.-C. and Wang, H.-J. (2004) Physical Review C, 70, Article ID: 044003. http://dx.doi.org/10.1103/PhysRevC.70.044003

[48] Arrington, J., Blunden, P.G. and Melnitchouk, W. (2011) Progress in Particle and Nuclear Physics, 66, 782-833. http://dx.doi.org/10.1016/j.ppnp.2011.07.003

[49] Cloet, I.C., Roberts, C.D. and Thomas, A.W. (2013) Physical Review Letters, 111, Article ID: 101803. http://dx.doi.org/10.1103/PhysRevLett.111.101803

[50] Callan Jr., C.G. (1970) Physical Review D, 2, 1541-1547. http://dx.doi.org/10.1103/PhysRevD.2.1541

[51] Symanzik, K. (1970) Communications in Mathematical Physics, 18, 227-246. http://dx.doi.org/10.1007/BF01649434

[52] Budini, P. (1979) Czechoslovak Journal of Physics B, 29, 6-21.

[53] Bateman, H. (1910) Proceedings of the London Mathematical Society, s2-8, 223-264. http://dx.doi.org/10.1112/plms/s2-8.1.223

[54] Cunningham, E. (1910) Proceedings of the London Mathematical Society, s2-8, 77-98. http://dx.doi.org/10.1112/plms/s2-8.1.77

[55] Polchinski, J. (1988) Nuclear Physics B, 303, 226-236. http://dx.doi.org/10.1016/0550-3213(88)90179-4

[56] Nakayama, Y. (2013) arXiv:1302.0884 [hep-th]. http://arxiv.org/abs/1302.0884

[57] Gross, D.J. and Wess, J. (1970) Physical Review D, 2, 753-764. http://dx.doi.org/10.1103/PhysRevD.2.753 
[58] Kastrup, H.A. (2008) Annalen der Physik, 17, 631-690. http://dx.doi.org/10.1002/andp.200810324

[59] Dirac, P.A.M. (1935) Annals of Mathematics, 37, 429-442. http://dx.doi.org/10.2307/1968455

[60] Luscher, M. and Mack, G. (1975) Communications in Mathematical Physics, 41, 203-234. http://dx.doi.org/10.1007/BF01608988

[61] Mack, G. and Salam, A. (1969) Annals of Physics, 53, 174-202. http://dx.doi.org/10.1016/0003-4916(69)90278-4

[62] Ryder, L.H. (1974) Journal of Physics A: Mathematical, Nuclear and General, 7, 1817-1828. http://dx.doi.org/10.1088/0305-4470/7/15/005

[63] Wheeler, J.A. and Feynman, R.P. (1945) Reviews of Modern Physics, 17, 157-181. (ibid, 21, 425 (1949))

[64] Budini, P., Furlan, P. and Raczka, R. (1979) IL Nuovo Cimento A, 52, 191-246. (21 Luglio 1979)

[65] Cartan, E. (1981) The Theory of Spinors. Dover Publications, Inc., Mineola. (This Is a Republication of the First Version Published by Hermann, Paris, 1966)

[66] Liu, Y.F., Ma, Z.Q. and Hou, B.Y. (1999) Communications in Theoretical Physics, 31, 481-490. http://dx.doi.org/10.1088/0253-6102/31/4/481

[67] Esteve, A. and Sona, P.G. (1964) IL Nuovo Cimento, 32, 473-485. http://dx.doi.org/10.1007/BF02733974

[68] Feynman, R.P. (1962) Quantum Electrodynamics-A Lecture Note and Preprint Volume. W. A. Benjamin, Inc., 44. (please refer to the Tenth Lecture on Equivalence Transformation)

[69] Mandl, F. and Shaw, G. (2010) Quantum Field Theory. 2nd Edition, John Wiley and Sons, Ltd., Hoboken. (page 93, Section 2.4 and Appendix A.7)

[70] Lurie, D. (1968) Particles and Fields. John Wiley and Sons, Inc., Hoboken. (Please refer to Eqs. (8.18) (8.29) and context around)

[71] Scalum turns out to be a new inner degree of freedom. Yukawa and Yennie [22] [23] once contemplated that there should appear new "inner motion" degree of freedom in a nonlocal system, later it proved to be trivial due to their wrongly interpreting manner.

[72] Leader, E. (2011) Physical Review D, 83, Article ID: 096012. http://dx.doi.org/10.1103/PhysRevD.83.096012

[73] Wang, F., Chen, X.S., Lu, X.F., Sun, W.M. and Goldman, T. (2008) Physical Review Letters, 100, Article ID: 232002.

[74] Chen, X.-S., Sun, W.-M., Wang, F. and Goldman, T. (2011) Physical Review D, 83, Article ID: 071901.

[75] Ji, X.D., Xiong, X.N. and Yuan, F. (2012) Physical Review Letters, 109, Article ID: 152005. http://dx.doi.org/10.1103/PhysRevLett.109.152005

[76] Ji, X.D. (1997) Physical Review D, 55, 7114-7125. http://dx.doi.org/10.1103/PhysRevD.55.7114

[77] Ji, X.D. (1997) Physical Review Letters, 78, 610-613. http://dx.doi.org/10.1103/PhysRevLett.78.610

[78] Ma, B.Q. (1991) Journal of Physics G, 17, L53-L58. http://dx.doi.org/10.1088/0954-3899/17/5/001

[79] Kodaira, J., Matsuda, S., Muta, T., Uematsu, T. and Sasaki, K. (1979) Physical Review D, 20, 627-629. http://dx.doi.org/10.1103/PhysRevD.20.627

[80] Jaffe, R.L. (1995) Physics Today, 48, 24. http://dx.doi.org/10.1063/1.881473

[81] Kodaira, J., Matsuda, S., Sasaki, K. and Uematsu, T. (1979) Nuclear Physics B, 159, 99-124. http://dx.doi.org/10.1016/0550-3213(79)90329-8

[82] Bass, S.D. (2005) Reviews of Modern Physics, 77, 1257-1302.

[83] Jaffe, R.L. and Manohar, A. (1990) Nuclear Physics B, 337, 509-546. http://dx.doi.org/10.1016/0550-3213(90)90506-9

[84] Myhrer, F. and Thomas, A.W. (2010) Journal of Physics G: Nuclear and Particle Physics, 37, Article ID: 023101. http://dx.doi.org/10.1088/0954-3899/37/2/023101

[85] de Florian, D., Sassot, R., Stratmann, M. and Vogelsang, W. (2008) Physical Review Letters, 101, Article ID: 072001.

[86] Chen, X.S., Lu, X.F., Sun, W.M., Wang, F. and Goldman, T. (2008) Physical Review Letters, 100, Article ID: 232002. http://dx.doi.org/10.1103/PhysRevLett.100.232002

[87] Chen, X.S., Sun, W.M., Lu, X.F., Wang, F. and Goldman, T. (2009) Physical Review Letters, 103, Article ID: 062001. http://dx.doi.org/10.1103/PhysRevLett.103.062001

[88] Lorcé, C. (2013) Physical Review D, 87, Article ID: 034031. http://dx.doi.org/10.1103/PhysRevD.87.034031

[89] Wakamatsu, M. (2010) Physical Review D, 81, Article ID: 114010. http://dx.doi.org/10.1103/PhysRevD.81.114010

[90] Wakamatsu, M. (2011) Physical Review D, 83, Article ID: 014012. http://dx.doi.org/10.1103/PhysRevD.83.014012

[91] Hatta, Y. (2011) Physical Review D, 84, Article ID: 041701. http://dx.doi.org/10.1103/PhysRevD.84.041701 
[92] Greiner, W., Schramm, S. and Stein, E. (2007) Quantum Chromodynamics. Third Revised and Enlarged Edition, Springer-Verlag, Berlin, Heidelberg and New York, 80-87.

[93] Su, J.C., Dong, Y.B. and Wu, S.S. (1992) Journal of Physics G: Nuclear and Particle Physics, 18, 1347-1354. http://dx.doi.org/10.1088/0954-3899/18/8/009

[94] Wang, H.-J. and Geng, W.T. (2007) Journal of Physics A: Mathematical and Theoretical, 40, 11617-11625. http://dx.doi.org/10.1088/1751-8113/40/38/012

[95] Blum, K. and Kleinpoppen, H. (1975) International Journal of Quantum Chemistry, 9, 415-424. http://dx.doi.org/10.1002/qua.560090851

[96] van Wyngaarden, W.L. and Walters, H.R.J. (1986) Journal of Physics B: Atomic and Molecular Physics, 19, 18271842. http://dx.doi.org/10.1088/0022-3700/19/12/021

[97] Kelsey, E.J. (1979) Physical Review A, 19, 1062-1066. http://dx.doi.org/10.1103/PhysRevA.19.1062

[98] Edmunds, P.W., McDowell, M.R.C. and van de Ree, J. (1983) Journal of Physics B: Atomic and Molecular Physics, 16, L453-L458. http://dx.doi.org/10.1088/0022-3700/16/15/006

[99] Burke, P.G. and Schey, H.M. (1962) Physical Review, 126, C163-C168. http://dx.doi.org/10.1103/PhysRev.126.163

[100] Ohmura, T., Hara, Y. and Yamanouchi, T. (1958) Progress of Theoretical Physics, 20, 82-88. http://dx.doi.org/10.1143/PTP.20.82

[101] Crowe, D.M., Guo, X.Q., Lubell, M.S., Slevin, J. and Eminyan, M. (1990) Journal of Physics B: Atomic, Molecular and Optical Physics, 23, L325-L331. http://dx.doi.org/10.1088/0953-4075/23/13/010

[102] Fletcher, G.D., Alguard, M.J., Gay, T.J., Hughes, V.W., Tu, C.W., Wainwright, P.F., et al. (1982) Physical Review Letters, 48, 1671-1674. http://dx.doi.org/10.1103/PhysRevLett.48.1671

[103] Williams, J.F. (1975) Journal of Physics B: Atomic and Molecular Physics, 8, 1683-1692. http://dx.doi.org/10.1088/0022-3700/8/10/018

[104] Gay, T.J., Fletcher, G.D., Alguard, M.J., Hughes, V.W., Wainwright, P.F. and Lubell, M.S. (1982) Physical Review A, 26, 3664-3667. http://dx.doi.org/10.1103/PhysRevA.26.3664

[105] Wang, H.-J. (2008) Journal of Mathematical Physics, 49, Article ID: 033513. http://dx.doi.org/10.1063/1.2874556

[106] Mastropietro, V. (2008) Non-Perturbative Renormalization. World Scientific Publishing Co. Pte. Ltd., Singapore.

[107] Berges, J., Tetradis, N. and Wetterich, C. (2002) Physics Reports, 363, 223-386. http://dx.doi.org/10.1016/S0370-1573(01)00098-9

[108] Wang, D. and Bao, A.D. (2013) Journal of Modern Physics, 4, 35-38. http://dx.doi.org/10.4236/jmp.2013.41007

[109] Greenberger, D.M. (1963) Annals of Physics, 25, 290-308. http://dx.doi.org/10.1016/0003-4916(63)90015-0

[110] Takahashi, Y. (1971) Physical Review D, 3, 622-625. http://dx.doi.org/10.1103/PhysRevD.3.622 